\title{
Rational Linker Design to Accelerate Excretion and Reduce Background Uptake of Peptidomimetic PSMA-Targeting Hybrid Molecules
}

Ann-Christin Eder ${ }^{1,2,3,4 *}$, Martin Schäfer ${ }^{1}$, Jana Schmidt ${ }^{1}$, Ulrike Bauder-Wüst ${ }^{1}$, Mareike Roscher $^{1}$, Karin Leotta ${ }^{4,5}$, Uwe Haberkorn ${ }^{4,5}$, Klaus Kopka ${ }^{1,6, \#, \dagger}$, Matthias Eder ${ }^{2,3, \dagger}$

†These authors contributed equally to the study and share last authorship.

1 Division of Radiopharmaceutical Chemistry, German Cancer Research Center (DKFZ), Heidelberg, Germany

2 Department of Nuclear Medicine, University Medical Center Freiburg, University of Freiburg, Faculty of Medicine, Freiburg, Germany

${ }^{3}$ Division of Radiopharmaceutical Development, German Cancer Consortium (DKTK), partner site Freiburg, Freiburg, Germany and German Cancer Research Center, Heidelberg, Germany

4 Department of Nuclear Medicine, Heidelberg University Hospital, Heidelberg, Germany

${ }^{5}$ Clinical Cooperation Unit Nuclear Medicine, German Cancer Research Center (DKFZ), Heidelberg, Germany

${ }^{6}$ German Cancer Consortium (DKTK), Heidelberg, Germany

\# current address: Helmholtz-Zentrum Dresden-Rossendorf (HZDR), Institute of Radiopharmaceutical Cancer Research, Dresden, Germany

* First and corresponding author:

Dr. Ann-Christin Eder

Department of Nuclear Medicine, University Medical Center Freiburg and Division of Radiopharmaceutical Development, German Cancer Consortium, partner site Freiburg, and German Cancer Research Center, Heidelberg, Germany Hugstetter Str. 55 79106 Freiburg

Tel: +4976127063370

Fax: +4976127039980

Email: ann-christin.eder@uniklinik-freiburg.de

Word count of the manuscript: 4996

Financial support: BMBF/VIP+ (VP00130)

Short running title:

Linker Design for PSMA Hybrid Molecules 


\section{ABSTRACT}

2 The evolution of peptidomimetic hybrid molecules for preoperative imaging and guided surgery

3 targeting the prostate-specific membrane antigen (PSMA) significantly progressed over the past

4 years and some approaches are currently evaluated for further clinical translation. However,

5 accumulation in non-malignant tissue such as kidney, bladder, spleen or liver might limit tumor-

6 to-background contrast for precise lesion delineation particularly in a surgical setting. To

7 overcome these limitations a rational linker design aims at the development of a second

8 generation of PSMA-11 based hybrid molecules with enhanced pharmacokinetic profile and

9 improved imaging contrasts. Methods: A selection of rational designed linkers was introduced to

10 the PSMA-targeting hybrid molecule Glu-urea-Lys-HBED-CC-IRDye800CW resulting in a

11 second generation peptidomimetic hybrid molecule library. The biological properties were

12 investigated in cell-based assays. In a preclinical proof-of-concept study with the radionuclide

$13{ }^{68} \mathrm{Ga}$, the impact of the modifications was evaluated by determination of specific tumor uptake,

14 pharmacokinetics and fluorescence-imaging in tumor-bearing mice. Results: The modified

15 hybrid molecules carrying various selected linkers revealed high PSMA-specific binding affinity

16 and effective internalization. The highest tumor-to-background contrast of all modifications

17 investigated was identified for the introduction of a histidine $(H)$ and glutamic acid $(E)$ containing

18 linker $\left((\mathrm{HE})_{3}\right.$-linker) between PSMA-binding motif and chelator. In comparison to the parental

19 core structure, uptake in non-malignant tissue was significantly reduced to a minimum

20 exemplified by an 11 -fold reduced spleen uptake from $38.12 \pm 14.62 \% \mathrm{ID} / \mathrm{g}$ to $3.47 \pm 1.39 \% \mathrm{ID} / \mathrm{g}(1$

21 h p.i.). The specific tumor uptake of this compound $(7.59 \pm 0.95 \% \mathrm{ID} / \mathrm{g}, 1 \mathrm{~h}$ p.i.) was detected to

22 be significantly higher as compared to the parental tracer PSMA-11. These findings confirmed

23 by PET and fluorescence imaging are accompanied by an enhanced pharmacokinetic profile

24 with accelerated background clearance at early time points post injection. Conclusion: The

25 novel generation of PSMA-targeting hybrid molecules reveal fast elimination, reduced

26 background organ enrichment and high PSMA-specific tumor uptake meeting the key demands 
27 for potent tracers in Nuclear Medicine and fluorescence-guided surgery. The approach's efficacy

28 of improving the pharmacokinetic profile highlights the strengths of rational linker design as a 29 powerful tool in strategic hybrid molecule development.

30

$31 \quad$ Key words

32 PSMA; hybrid molecules; prostate cancer; guided-surgery; pharmacokinetic profile 


\section{INTRODUCTION}

34 Surgical resection of tumor tissue represents one of the main curative treatment options in the

35 clinical management of prostate cancer (1). The precise detection and comprehensive resection

36 of malignancies is thereby of high significance for patient survival and therapy success. During

37 resection several difficulties are limiting the therapeutic outcome. While malignant tissue can be

38 precisely localized preoperatively using diagnostic radiopharmaceuticals, the translation to the

39 operating theatre often remains challenging. This increases the risk of tumor tissue being missed

40 out by the surgeon (2,3). Additionally, a close proximity of lesions to essential healthy structures

41 such as urinary bladder or nerves impedes a wide dissection in the lower pelvis resulting in

42 positive surgical margins (4). These difficulties cause an increased possibility of cancer

43 recurrence and subsequent treatment failure (5). Consequently, for example template-based

44 extended lymphadenectomies are performed to diminish the risk of left behind lesions $(6,7)$.

45 Besides malignant tissue, a considerable amount of healthy tissue is removed during this

46 surgical strategy causing an increased morbidity. Hence, there is a strong medical need for

47 advances in the field of intraoperative navigation to precisely delineate tumor tissue from

48 surrounding healthy tissue.

To overcome these issues, novel approaches have been developed over the past years

50 comprising the detection of malignant tissue supported by both a gamma probe and a

51 fluorescent dye. A combination of these two modalities in so-called hybrid or dual-labeled

52 approaches compensates for the respective disadvantages thereby merging the strength of both

53 technologies. In a clinical scenario, these novel approaches provide preoperative imaging (e.g.

$54 \mathrm{PET} / \mathrm{CT}$ ) for planning of the surgery combined with subsequent intraoperative navigation. First

55 clinical proof-of-concept studies with the indocyanine green (ICG)-based hybrid sentinel lymph

56 node tracer ICG-99mTc-NanoColloid demonstrated the feasibility of dual-modality approaches to

57 improve surgical accuracy in different cancer types (8-10). As non-targeted approaches have 
58 their limitations to precisely detect tumor tissue, recent advances focus on the design of targeted 59 dual-modality probes.

For the specific targeting of prostate cancer, the prostate-specific membrane antigen 61 (PSMA) has been identified as an excellent target structure. PSMA is a transmembrane 62 carboxypeptidase, which is selectively overexpressed in the majority of prostate carcinomas, 63 including local lesions, malignant lymph nodes and bone metastases (11-14). First PSMA64 targeting dual-modality antibodies and small molecule peptidomimetic inhibitors have recently demonstrated their feasibility of hybrid detection in preclinical studies (15-18). Besides high and specific tumor uptake of targeted hybrid probes, a favorable pharmacokinetic profile with e.g. low accumulation in off-target tissue, fast clearance and a resulting high imaging contrast at early time points post injection is crucial for further clinical translation.

With the development of dual-modality low molecular weight PSMA-inhibitors based on

70 the clinically established PET-tracer ${ }^{68} \mathrm{Ga}-\mathrm{PSMA}-11$, a versatile platform was designed tolerating 71 the conjugation of a fluorescent dye combined with a radiolabel moiety (18-20). Our theranostic 72 dual-modality platform is thus characterized by high and fast PSMA-specific tumor uptake along 73 with rapid background clearance allowing preoperative imaging combined with intraoperative 74 guidance (18). The advantageous effect of introducing spacer moieties comprising histidine $(\mathrm{H})$ 75 and glutamic acid (E) on the biodistribution profile of affibodies was originally reported by 76 Hofstrom et al. (21). Further work from our group successfully established histidine/glutamic acid 77 linker modifications to PSMA-11 leading to significantly enhanced tumor-to-background contrast 78 and reduced uptake in dose limiting background organs (22). Due to the high clinical relevance, 79 we introduced charged spacer moieties to our theranostic dual-modality platform leading to a 80 second generation of hybrid probes with improved imaging contrasts. The insights investigated 81 in this pharmacokinetic proof-of-concept study, demonstrate a valuable progression aiming at 82 clinical translation for better management of prostate cancer patients. 

91 the Supplemental Data (26).

92

\section{METHODS}

\section{In Vitro Evaluation}

\section{Chemical Synthesis, Radiolabeling, Determination of Lipophilicity and Serum Stability}

The synthesis of the variants of Glu-urea-Lys-HBED-CC-IRDye800CW comprising a series of amino acid linker modifications was performed according to previously published protocols $(18,22-25)$ (see Supplemental Data for details on the synthesis and chemical structures of the compounds). ${ }^{68} \mathrm{Ga}^{3+}$ (half-life $68 \mathrm{~min} ; \beta^{+} 89 \%$; $\mathrm{E}_{\beta^{+}}$max. $1.9 \mathrm{MeV}$ ) was obtained from a ${ }^{68} \mathrm{Ge} /{ }^{68} \mathrm{Ga}$ generator based on a pyrogallol resin support with details of compound characterization (radiolabeling, determination of lipophilicity, serum stability studies) provided in

PSMA-positive LNCaP cells (CRL-1740; ATCC) and PSMA-negative PC-3 cells (CRL1435; ATCC) were cultured in RPMI medium supplemented with $10 \%$ fetal calf serum and 2 $\mathrm{mmol} / \mathrm{L}$ L-glutamine (all from PAA). Cells were grown at $37^{\circ} \mathrm{C}$ in humidified air with $5 \% \mathrm{CO}_{2}$ and were harvested using trypsin-ethylenediaminetetraacetic acid (trypsin-EDTA; $0.25 \%$ trypsin, $0.02 \%$ EDTA, Invitrogen). Cell line authentication is regularly performed and the authentication of the LNCaP and PC-3 cell lines was confirmed on 03/06/2020. The competitive cell binding assay and internalization experiments were performed as described previously $(19,25)$.

\section{Biodistribution and Preclinical Proof-of-Concept}

For the experimental tumor models $5 \times 10^{6}$ cells of LNCaP or PC-3 (in 50\% Matrigel; Becton Dickinson) were subcutaneously inoculated into the flank of 7 - to 8-week-old male BALB/c nu/nu mice (Charles River). For biodistribution studies, the ${ }^{68} \mathrm{Ga}$-labeled compounds were injected into a tail vein (1-3 MBq; 60 pmol, $\mathrm{n}=3$ and 30-50 MBq; 500 pmol, $\mathrm{n}=3$ ). At $1 \mathrm{~h}$ and $2 \mathrm{~h}$ after injection, respectively, the animals were sacrificed. Organs of interest were dissected, blotted dry, and weighed. The radioactivity was measured using a gamma counter and calculated as \% ID/g. 
109 Optical imaging was performed with the Odyssey CLx system (LI-COR Biosciences, excitation 110 wavelength $800 \mathrm{~nm})$. In an additional preclinical proof-of-concept $(\mathrm{n}=1)$, mice were anesthetized 111 (2\% sevoflurane; Abbvie) and $0.5 \mathrm{nmol}$ of the ${ }^{68} \mathrm{Ga}$-labeled compound in $0.9 \% \mathrm{NaCl}(\mathrm{pH} 7)$ were 112 injected into the tail vein. Preoperative PET imaging was performed with a PET scanner (Inveon 113 PET; Siemens). For subsequent optical imaging to identify the tumor by fluorescence imaging, 114 mice were sacrificed after PET imaging and dissected tissue analyzed using the Odyssey CLx 115 system (see Supplemental Data for details on the imaging protocol, software, image 116 reconstruction and procedure). All animal experiments were approved by the regional authorities 117 Regierungspräsidium Karlsruhe and Regierungspräsidium Freiburg and complied with the 118 current laws of the Federal Republic of Germany.

\section{Statistical Aspects}

Experiments were performed at least in triplicate except the proof-of-concept study $(n=1)$.

122 Quantitative data are expressed as mean \pm SD. The $n$-values are given in the respective figure 123 or table captions. If applicable, means were compared using Student's $t$ test (GraphPad Prism

124 Version 8, GraphPad Software, Inc.). p-values < 0.05 were considered statistically significant. 


\section{Synthesis, Radiolabeling and Serum Stability}

For this pharmacokinetic proof-of-concept study, Glu-urea-Lys-HBED-CC-IRDye800CW

(tumor uptake $1 \mathrm{~h}$ p.i.: $13.66 \pm 3.73 \% \mathrm{ID} / \mathrm{g}$ ) was selected as exemplary model core structure of our theranostic dual-modality platform for two main reasons. First, this compound has been preclinically proven to perform comparably to or even outperformed successfully established molecules such as PSMA-11 (tumor uptake 1 h p.i.: $4.89 \pm 1.34 \%$ ID/g) or PSMA-617 (tumor uptake 1 h p.i.: $8.47 \pm 4.09 \% \mathrm{ID} / \mathrm{g})(18,22,27)$. Second, the choice of the structure previously comprising the bulky clinically relevant NIR-dye IRDye800CW simplifies further clinical translation of the findings, since small molecules' biological properties are typically strongly influenced when conjugated to a fluorescent dye. In a first step, charged linker moieties identified favourable with respect to pharmacokinetic properties in previous studies were 137 introduced combining solid phase and classical organic synthesis strategies (Supplemental Figs.

1381 and 2$)(18,22)$. As the most promising candidate in former studies, the $(\mathrm{HE})_{3}$-motif was 139 additionally inserted between chelator and fluorescent moiety to investigate the influence of 140 linker positioning within the molecule (Supplemental Fig. 2) $(23,28)$. The dye was conjugated to 141 the precursor molecules as IRDye800CW-NHS-ester in the last step. A detailed description of 142 the synthetic strategies is provided in the Supplemental Information. The final products were 143 obtained in $>98 \%$ purity and their analytical data is summarized in the Supplemental Data 144 (Supplemental Figs. 3 and 4; Supplemental Tables 1 and 2). Lipophilicity determined as $\log D$ at $\mathrm{pH} 7.4$ in n-octanol/PBS was found to be in the same range compared to the respective Gluurea-Lys-HBED-CC-IRDye800CW reference compound with a $\log D_{\mathrm{pH}} 7.4$ value of $-2.21 \pm 0.36$ 147 (Supplemental Table 1) (18). Radiolabeling with ${ }^{68} \mathrm{Ga}$ resulted in radiochemical yields $>99 \%$ and 148 the molar activities of the ${ }^{68} \mathrm{Ga}$-labeled compounds were detected to be around 80 $149120 \mathrm{GBq} / \mu \mathrm{mol}$ (Supplemental Figs. 5-7). ${ }^{68} \mathrm{Ga}-$ Glu-urea-Lys-(HE)) - HBED-CC-IRDye800CW was 150 found to be stable in mouse and human serum up to $2 \mathrm{~h}$. 
High and specific affinity to PSMA in the nanomolar range was revealed for all tested compounds in competitive binding studies, which additionally was found to be not significantly complexation-dependent (Table 1; Supplemental Fig. 8). The introduction of $(\mathrm{HE})_{1-}$ or trypotophane (W) containing (WE) 1 -motifs between PSMA-binding motif and chelator or the $156(\mathrm{HE})_{3}$-motif between chelator and fluorescent dye had no impact on binding properties as 157 compared to the reference Glu-urea-Lys-HBED-CC-IRDye800CW (18). Interestingly, the $(\mathrm{HE})_{3^{-}}$ 158 motif inserted between PSMA binding unit and chelator moiety significantly reduced the affinity 159 to PSMA ( $p=0.024)$. Specific cell surface binding was detected for all tested dual-labeled probes

160 (Table 1; Supplemental Fig. 9). Incorporating the $(\mathrm{WE})_{1-}(\mathrm{p}=0.482)$ or $(\mathrm{HE})_{3}-\mathrm{motif}(p=0.053)$ in 161 close proximity to the PSMA-binding motif resulted in the most pronounced specifically 162 internalized fractions not significantly differing from the reference (18). In contrast, changing the 163 intramolecular $(\mathrm{HE})_{3}$-motif position by inserting the linker between chelator and dye $(p=0.021)$ or 164 introducing $(\mathrm{HE})_{1}$ as a linker structure next to the PSMA-binding motif $(p=0.021)$ revealed 165 significantly reduced internalization properties (Table 1).

\section{Specific Enrichment in Xenograft Tumors with Enhanced Contrast to Background Tissue}

The impact of the introduction of charged amino acid linker motifs to Glu-urea-Lys-HBEDCC-IRDye800CW on in vivo specific PSMA targeting and accompanying background organ enrichment was further evaluated in biodistribution studies. ${ }^{68} \mathrm{Ga}-\mathrm{Glu}$-urea-Lys-(HE) ${ }_{3}$-HBED-CC-

171 IRDye800CW revealed a significant higher, PSMA-specific tumor uptake in LNCaP xenograft 172 tumors $(7.59 \pm 0.95 \% \mathrm{ID} / \mathrm{g}, \mathrm{p}<0.05)$, compared to the incorporation of other motifs or 173 intramolecular positioning of the $(\mathrm{HE})_{3}$-motif in closer proximity to the fluorescent dye at $1 \mathrm{~h}$ post 174 injection (p.i.) (Fig. 1A, B; Supplemental Fig. 10; Supplemental Tables 3-5). Although in 175 comparison to the core structure, tumor uptake was significantly reduced for ${ }^{68} \mathrm{Ga}-\mathrm{Glu}$-urea-Lys$176(\mathrm{HE})_{3}$-HBED-CC-IRDye800CW ( $\left.\mathrm{p}=0.031\right)$, it proved to be significantly higher when compared to 
${ }^{68} \mathrm{Ga}-\mathrm{PSMA}-11(\mathrm{p}=0.047)$ or ${ }^{68} \mathrm{Ga}-\mathrm{PSMA}-\mathrm{I} \& \mathrm{~F}(\mathrm{p}=0.045)$, and identical as compared to ${ }^{68} \mathrm{Ga}-$ PSMA-617 $(p=0.735)(17,22,27)$. In addition, tracer uptake of ${ }^{68} \mathrm{Ga}-G l u-u r e a-L y s-(H E)_{3}-H B E D-$ CC-IRDye800CW in non-malignant tissue was significantly reduced to a minimum with striking effects on e.g. spleen uptake, which could be reduced from $38.12 \pm 14.62 \%$ ID/g to $3.47 \pm 1.39$ \%ID/g (Fig. 1A; Supplemental Table 3) (18). From the here presented series, the introduction of 182 the $(\mathrm{HE})_{3}$-motif in close proximity to the PSMA-binding motif led to the highest tumor-to183 background contrast. Noticeably, this compound even outperforms the core structure Glu-urea184 Lys-HBED-CC-IRDye800CW with regard to tumor-to-background ratio (Supplemental Table 4). 185 For all compounds the renal pathway was identified to be the most likely elimination mechanism.

Additionally, to finalize the analysis of the pharmacokinetic properties of the favoured compound ${ }^{68} \mathrm{Ga}-$ Glu-urea-Lys-(HE) $)_{3}$-HBED-CC-IRDye800CW, biodistribution studies at $2 \mathrm{~h}$ p.i. were conducted (Fig. 1C, D; Supplemental Table 6). Specific tumor accumulation was significantly reduced after injection of $60 \mathrm{pmol}(3.10 \pm 1.17 \% \mathrm{ID} / \mathrm{g})$ as compared to $1 \mathrm{~h}$ p.i. $(7.59 \pm 0.95 \% \mathrm{ID} / \mathrm{g})(\mathrm{p}=0.007)$. Contemporaneously, the compound was strongly excreted from background tissue resulting in remaining high tumor-to-background ratios up to $2 \mathrm{~h}$ p.i. 192 (Supplemental Table 6). Administration of higher doses of ${ }^{68} \mathrm{Ga}-\mathrm{Glu}$-urea-Lys-(HE) $)_{3}-\mathrm{HBED}-\mathrm{CC}$ 193 IRDye800CW (500 pmol) mainly confirmed the findings and even results in higher tumor-to194 background ratios for muscle 1 and 2 h p.i. (Fig. 1C, D; Supplemental Tables 7 and 8 ).

\section{Small-Animal PET and Optical Imaging}

Subsequent small-animal PET studies with ${ }^{68}$ Ga-Glu-urea-Lys-(HE) ${ }_{3}$-HBED-CCIRDye800CW confirmed strong tumor uptake in the LNCaP xenograft model accompanied by rapid clearance from non-malignant tissue resulting in high imaging contrasts at early time points

200 p.i. (Fig. 2 A-D). High PSMA-specificity was proven in vivo as no measurable uptake was 201 observed in PSMA-negative PC-3 xenografts (Fig. 2 B). The corresponding time activity curves 202 of the dynamic PET scan up to 60 min p.i. demonstrate rapid clearance from off-target tissue 
203 (muscle, liver, heart), but continuous accumulation in bladder and kidney due to the renal 204 pathway of excretion (Fig. 2 C).

In fluorescence imaging ${ }^{68}$ Ga-labeled Glu-urea-Lys-(HE) ${ }_{3}-H B E D-C C-I R D y e 800 C W$ obtained high tumor uptake resulting in excellent tumor visualization and strong tumor-tobackground contrasts (Fig. 3; Supplemental Figs. 11 and 12). Additionally, the renal excretion pathway and the significantly reduced spleen uptake detected based on radioactivity was fluorescently confirmed.

\section{DISCUSSION}

The development of dual-modality probes for preoperative imaging and intraoperative 213 (radio- or fluorescence-) guidance specifically targeting PSMA represents a promising new 214 strategy in the diagnosis and therapy of PCa. The improved detection of tumor tissue supported 215 by both, a gamma signal and a fluorescent dye, might overcome current surgical limitations. 216 Therefore, a novel class of dual-labeled low molecular weight PSMA inhibitors has recently been developed thereby successfully demonstrating their feasibility for clinical translation in preclinical

218 proof-of-concept studies (16-18). In particular, a specific and sufficiently high tumor uptake 219 combined with a fast pharmacokinetic profile resulting in high tumor-to-background contrasts at 220 early time points post injection challenges low molecular weight hybrid tracer development. $221{ }^{68}$ Ga-Glu-urea-Lys-HBED-CC-IRDye800CW was the first PSMA-targeting low molecular weight 222 hybrid probe characterized by a 3-fold increased tumor uptake compared to the parental 223 molecule PSMA-11, while preserving a fast pharmacokinetic (PK) profile.

Nevertheless, like other peptidomimetic PSMA inhibitors this dual-modality approach 225 results in excretion via the renal pathway and accumulation in non-malignant tissue (spleen). 226 Probe enrichment close to the surgical field might hamper specific lesion detection in particular 
close to the urinary system. Additionally, non-malignant accumulation might be of concern 228 regarding toxicity issues during clinical translation of this approach.

To address this limitation, our study discovers rational hybrid molecule design as a promising tool to accelerate the PK profile accompanied by a preserved high tumor uptake. In 231 particular, introduction of spacer moieties comprising histidine $(\mathrm{H})$ and glutamic acid $(\mathrm{E})$ proved 232 favourable to modulate the biodistribution profile of affibodies and petidomimetic inhibitors 233 thereby improving tumor-to-background contrast and potential uptake in dose limiting non234 malignant tissue (21-23).

In the here presented study, rational linker design is applied on the peptidomimetic hybrid 236 molecule Glu-urea-Lys-HBED-CC-IRDye800CW, the current lead of our theranostic dual237 modality platform. Due to its favourable characteristics (high specific tumor uptake, fast PK 238 properties) and successfully incorporated clinically relevant NIR-dye (simplified clinical 239 translation of PK study findings), it was designated for a further proof-of-concept PK-modulation 240 (18). The chemical motifs $(\mathrm{HE})_{1},(\mathrm{HE})_{3}$ and $(\mathrm{WE})_{1}$ found to be beneficial in previous studies were 241 selected for this approach (21-23).

Binding affinities of all second generation compounds resulting from the present study 243 were in the low nanomolar range indicating negligible functional impact of the selected linker 244 motifs. Nevertheless, inserting the (HE)-motif as a triplicate between PSMA binding motif and 245 chelator slightly reduces PSMA affinity. While a single charged and/or lipophilic motif is tolerated 246 in terms of affinity, motif repetitions might hamper the advantageous interactions of the chelator 247 HBED-CC with the arene binding side of the PSMA binding pocket (29). Specific internalization 248 of all modifications was observed to be highest for the tryptophan-containing motif located close 249 to the PSMA-binding motif, hypothetically exploiting supportive lipophilic interactions in the 250 PSMA funnel (30). 

the PSMA binding unit and the radiometal chelator significantly enhanced tumor uptake in 254 previous findings (22), the effect could not be demonstrated in this study. Strikingly, including the $255(\mathrm{HE})_{3}$-motif at the same intramolecular location resulted in a strongly improved background 256 organ enrichment profile of the hybrid molecule at early time points post injection (1 h p.i.). In 257 particular, spleen uptake was reduced by a factor of 11 when compared to the parental structure $258{ }^{68} \mathrm{Ga}-$-Glu-urea-Lys-HBED-CC-IRDye800CW matching findings of earlier studies (22). In addition, 259 this modification revealed the highest tumor-to-background ratios to relevant non-malignant 260 tissue of all tested compounds including the parental structure. The resulting tumor uptake was 261 significantly higher as compared to all the other modified variants of this study, including PSMA26211 and PSMA-I\&F, however not exceeding the core structure Glu-urea-Lys-HBED-CC263 IRDye800CW and PSMA-617 (17,19,27). Accordingly, it can be concluded that the novel 264 compound features superior characteristics in terms of tumor uptake and tumor-to-background 265 contrast warranting further investigation.

Thus, the exact intramolecular position of $(\mathrm{HE})_{3}$ was found to be highly crucial, as an 267 introduction on a different location in the molecule results in significantly reduced or even 268 negative effects on background organ enrichment and tumor uptake. The introduction of $(\mathrm{HE})_{3}$ in 269 close proximity to the PSMA binding motif was found to noticeably influence for instance the 270 spleen uptake, which is in line with previous studies (22).

272 Glu-urea-Lys-(HE) $)_{3}$-HBED-CC-IRDye800CW was accompanied by a significantly accelerated 273 excretion via the renal pathway by means of reduced kidney enrichment as compared to the 274 parental structure ${ }^{68} \mathrm{Ga}-G l u-u r e a-L y s-H B E D-C C-I R D y e 800 \mathrm{CW}$ leading to high tumor-to275 background-contrast as early as $1 \mathrm{~h}$ p.i., which proved persistent up to $2 \mathrm{~h}$ p.i. (18). Surprisingly, 
276 at $1 \mathrm{~h}$ p.i., the PK-improved compound showed an about 60 minutes earlier manifestation of the

277 high tumor-to-background contrast as compared to the parental compound. This is caused by a

278 dramatically minimized enrichment in non-malignant tissue leading to 2.0-fold higher tumor-to-

279 blood and 1.6-fold higher tumor-to-muscle ratio at early time points after injection of 60 pmol.

280 Consequently, an increased contrast in the surgical field can be expected from these data 281 encouraging further clinical translation. Since other dyes are also being discussed in terms of 282 e.g. stability, brightness or tissue penetration, further studies might identify the ideal combination 283 finally representing the lead second generation PSMA-targeting hybrid probe.

\section{CONCLUSION}

This study discovers rational linker design as a powerful tool in hybrid molecule development leading to a novel generation of PSMA-targeting peptidomimetic hybrid molecules with significantly improved PK profile. With fast elimination and subsequent reduced enrichment in non-malignant tissue, this approach addresses the highly disruptive factor of background accumulation during surgical resection, while preserving a high PSMA-specific tumor uptake.

291 The PK modification strategy uncovered in this study, offers extensive utility in the future 292 discovery and development of targeted peptidomimetic hybrid agents.

\section{DISCLOSURE}

MS, UBW, UH, ME, KK, ACE hold patent rights on dual-labeled PSMA inhibitors. All other authors declare no competing interests.

\section{ACKNOWLEDGEMENTS}

We gratefully acknowledge support by the VIP+ grant VP00130, Federal Ministry of 300 Education \& Research (BMBF), Germany. 


\section{KEY POINTS}

302 Question

303 Is the tool of rational linker design suitable for peptidomimetic hybrid molecule 304 development to improve the pharmacokinetic profile and elimination rate?

306 Pertinent Findings

This preclinical proof-of-concept study discovers rational linker design as a valuable tool 308 to selectively affect the pharmacokinetic profile and elimination rate of PSMA-targeted 309 peptidomimetic hybrid molecules. By introducing a designated sequence of amino acids, 310 enrichment in non-malignant tissue was significantly reduced to a minimum thereby also 311 enhancing the elimination profile, while a high specific tumor uptake was preserved.

\section{Implications for Patient Care}

314 Background organ accumulation of novel hybrid molecules in e.g. the surgical field 315 represents one of the main challenges in hybrid molecule development. The study findings 316 introduce a tool to overcome these limitations and further advance discovery in novel image317 guided surgery approaches for clinical translation. 
1. Cornford $P$, van den Bergh RCN, Briers E, et al. EAU-EANM-ESTRO-ESUR-SIOG Guidelines on prostate cancer. Part II-2020 Update: Treatment of relapsing and metastatic prostate cancer. Eur Urol. 2020.

2. Weckermann D, Dorn R, Trefz M, Wagner T, Wawroschek F, Harzmann R. Sentinel lymph node dissection for prostate cancer: experience with more than 1,000 patients. J Urol. 2007;177:916-920.

3. Mattei A, Fuechsel FG, Bhatta Dhar N, et al. The template of the primary lymphatic landing sites of the prostate should be revisited: results of a multimodality mapping study. Eur Urol. 2008;53:118-125.

4. Yossepowitch O, Briganti A, Eastham JA, et al. Positive surgical margins after radical prostatectomy: a systematic review and contemporary update. Eur Urol. 2014;65:303-313.

5. Stephenson AJ, Eggener SE, Hernandez AV, et al. Do margins matter? The influence of positive surgical margins on prostate cancer-specific mortality. Eur Urol. 2014;65:675-680.

6. Munbauhal G, Seisen T, Gomez FD, et al. Current perspectives of sentinel lymph node dissection at the time of radical surgery for prostate cancer. Cancer Treat Rev. 2016;50:228-239.

7. Kiss B, Thoeny HC, Studer UE. Current status of lymph node imaging in bladder and prostate cancer. Urology. 2016;96:1-7.

8. van der Poel HG, Buckle T, Brouwer OR, Valdes Olmos RA, van Leeuwen FW. Intraoperative laparoscopic fluorescence guidance to the sentinel lymph node in prostate cancer patients: clinical proof of concept of an integrated functional imaging approach using a multimodal tracer. Eur Urol. 2011;60:826-833.

9. Brouwer OR, Buckle T, Vermeeren $\mathrm{L}$, et al. Comparing the hybrid fluorescent-radioactive tracer indocyanine green-99mTc-nanocolloid with $99 \mathrm{mTc}$-nanocolloid for sentinel node identification: a validation study using lymphoscintigraphy and SPECT/CT. J Nucl Med. 2012;53:1034-1040.

10. KleinJan GH, van den Berg NS, de Jong J, et al. Multimodal hybrid imaging agents for sentinel node mapping as a means to (re)connect nuclear medicine to advances made in robot-assisted surgery. Eur J Nucl Med Mol Imaging. 2016;43:1278-1287. expression in normal and malignant human tissues. Clin Cancer Res. 1997;3:81-85. 
12. Wright GL, Jr., Haley C, Beckett ML, Schellhammer PF. Expression of prostate-specific membrane antigen in normal, benign, and malignant prostate tissues. Urol Oncol. 1995;1:18-28.

13. Sweat SD, Pacelli A, Murphy GP, Bostwick DG. Prostate-specific membrane antigen expression is greatest in prostate adenocarcinoma and lymph node metastases. Urology. 1998;52:637-640.

14. Minner S, Wittmer C, Graefen M, et al. High level PSMA expression is associated with early psa recurrence in surgically treated prostate cancer. Prostate. 2011;71:281-288.

15. Lutje S, Rijpkema M, Franssen GM, et al. Dual-modality image-guided surgery of prostate cancer with a radiolabeled fluorescent anti-PSMA monoclonal antibody. J Nucl Med. 2014;55:995-1001.

16. Banerjee SR, Pullambhatla $M$, Byun $Y$, et al. Sequential SPECT and optical imaging of experimental models of prostate cancer with a dual modality inhibitor of the prostate-specific membrane antigen. Angew Chem Int Ed Engl. 2011;50:9167-9170.

17. Schottelius $M$, Wurzer A, Wissmiller $K$, et al. Synthesis and preclinical characterization of the PSMA-targeted hybrid tracer PSMA-I\&F for nuclear and fluorescence imaging of prostate cancer. J Nucl Med. 2019;60:71-78.

18. Baranski AC, Schafer M, Bauder-Wust U, et al. PSMA-11-derived dual-labeled PSMA inhibitors for preoperative PET imaging and precise fluorescence-guided surgery of prostate cancer. J Nucl Med. 2018;59:639-645.

19. Eder M, Schafer M, Bauder-Wust $\mathrm{U}$, et al. 68Ga-complex lipophilicity and the targeting property of a urea-based PSMA inhibitor for PET imaging. Bioconjug Chem. 2012;23:688-697.

20. Hope TA, Aggarwal R, Chee B, et al. Impact of (68)Ga-PSMA-11 PET on management in patients with biochemically recurrent prostate cancer. J Nucl Med. 2017;58:1956-1961.

21. Hofstrom C, Orlova A, Altai M, Wangsell F, Graslund T, Tolmachev V. Use of a HEHEHE purification tag instead of a hexahistidine tag improves biodistribution of affibody molecules sitespecifically labeled with (99m)Tc, (111)In, and (125)I. J Med Chem. 2011;54:3817-3826.

22. Baranski AC, Schafer M, Bauder-Wust U, et al. Improving the imaging contrast of (68)Ga-PSMA11 by targeted linker design: charged spacer moieties enhance the pharmacokinetic properties. 393 Bioconjug Chem. 2017;28:2485-2492.

23. Eder $M$, Lohr $T$, Bauder-Wust $U$, et al. Pharmacokinetic properties of peptidic radiopharmaceuticals: reduced uptake of (EH)3-conjugates in important organs. J Nucl Med. 2013;54:1-4. 
24. Eder M, Wangler B, Knackmuss $\mathrm{S}$, et al. Tetrafluorophenolate of HBED-CC: a versatile conjugation agent for 68Ga-labeled small recombinant antibodies. Eur J Nucl Med Mol Imaging. 2008;35:1878-1886.

400

25. Schafer $\mathrm{M}$, Bauder-Wust $\mathrm{U}$, Leotta $\mathrm{K}$, et al. A dimerized urea-based inhibitor of the prostatespecific membrane antigen for 68Ga-PET imaging of prostate cancer. EJNMMI Res. 2012;2:23.

403

26. Schuhmacher J, Maier-Borst W. A new Ge-68/Ga-68 radioisotope generator system for production of Ga-68 in dilute $\mathrm{HCl}$. Int J Appl Radiat Isot. 1981;32:31-36.

406

407

408

409

410

411

412

413

414

415

416

417

418

419

420

421

422
27. Benesova $M$, Schafer $M$, Bauder-Wust $U$, et al. Preclinical evaluation of a tailor-made DOTAconjugated PSMA inhibitor with optimized linker moiety for imaging and endoradiotherapy of prostate cancer. J Nucl Med. 2015;56:914-920.

28. Liolios C, Schafer M, Haberkorn U, Eder M, Kopka K. Novel bispecific PSMA/GRPr targeting radioligands with optimized pharmacokinetics for improved PET imaging of prostate cancer. Bioconjug Chem. 2016;27:737-751.

29. Zhang $A X$, Murelli RP, Barinka $C$, et al. A remote arene-binding site on prostate specific membrane antigen revealed by antibody-recruiting small molecules. J Am Chem Soc. 2010;132:1271112716.

30. Mesters JR, Barinka C, Li W, et al. Structure of glutamate carboxypeptidase II, a drug target in neuronal damage and prostate cancer. EMBO J. 2006;25:1375-1384. 
A

- ${ }^{68}$ Ga-Glu-urea-Lys-(HE) $)_{3}$-HBED-CC-IRDye800CW

$\square{ }^{68} \mathrm{Ga}$-Glu-urea-Lys-(HE) ${ }_{1}$-HBED-CC-IRDye800CW

$\square{ }^{68}$ Ga-Glu-urea-Lys-(WE) 1 -HBED-CC-IRDye800CW

ZU ${ }^{68} \mathrm{Ga}$-Glu-urea-Lys-HBED-CC-(HE) $)_{3}$-IRDye $800 \mathrm{CW}$
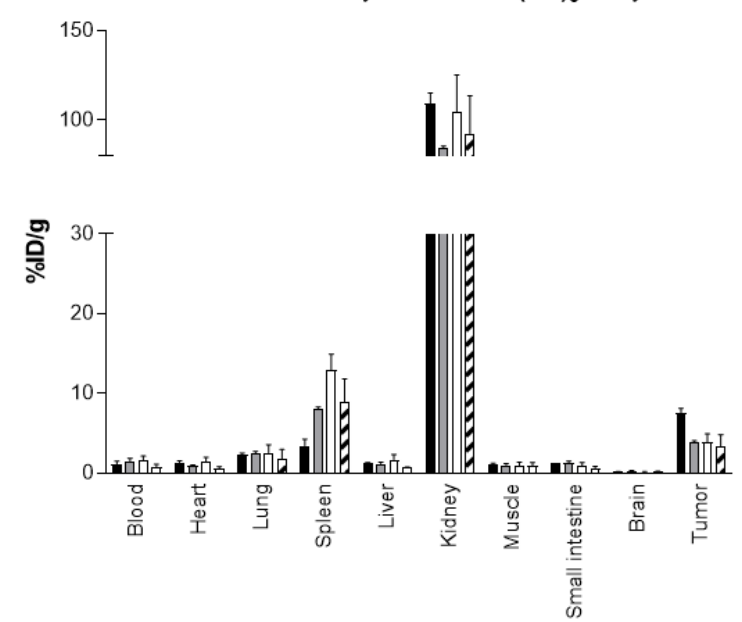

C

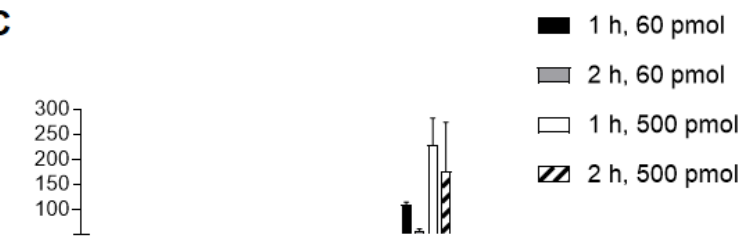

424

425

426

427

428

429

430
B

- LNCaP

$\square$ PC-3

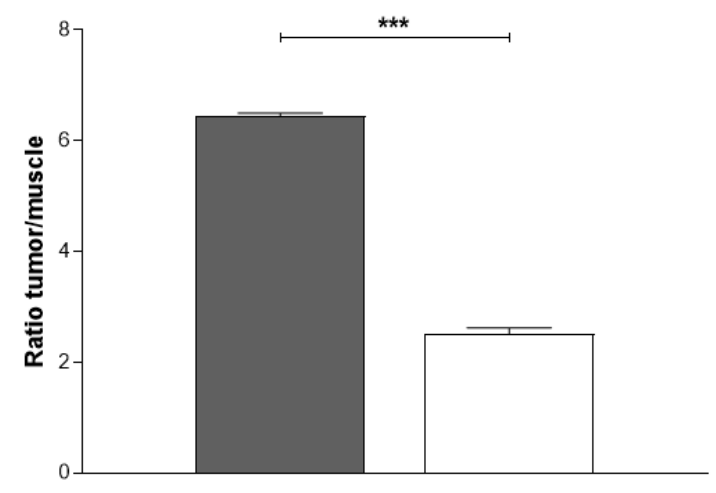

D

- $1 \mathrm{~h}, 60 \mathrm{pmol}$

घ $2 \mathrm{~h}, 60 \mathrm{pmol}$

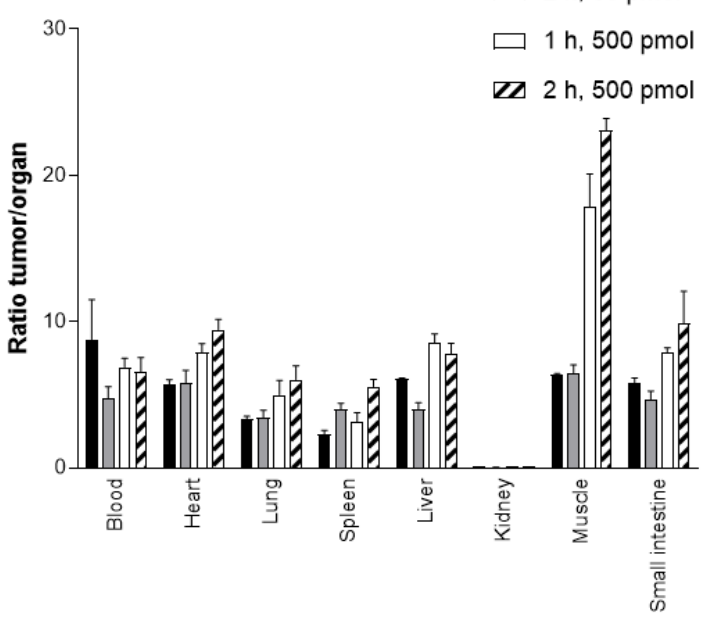



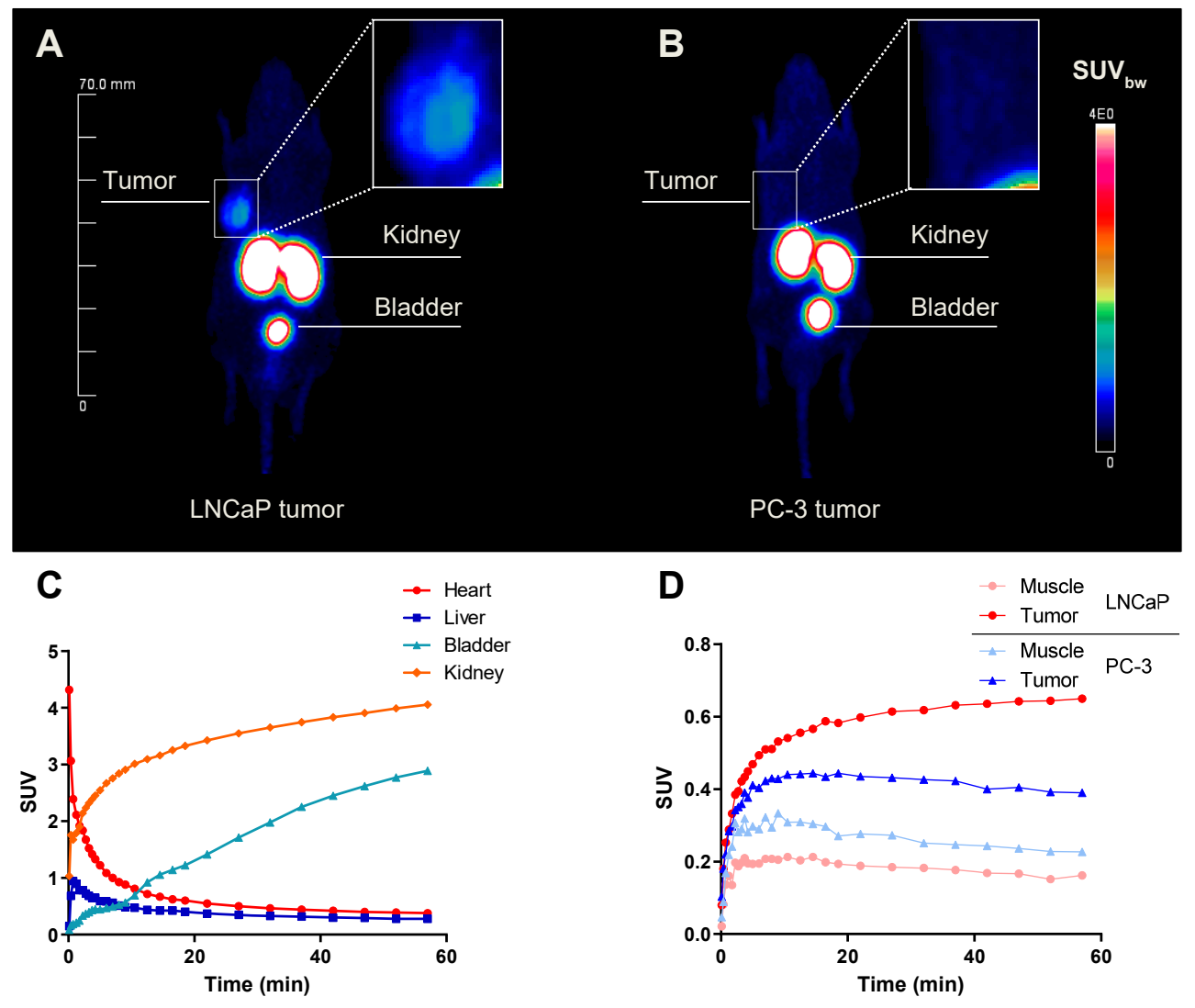

Figure 2. Small-animal PET imaging study. Whole-body maximum intensity projections with tumor magnifications of $4480.5 \mathrm{nmol}{ }^{68} \mathrm{Ga}-$ labeled Glu-urea-Lys-(HE) 3 -HBED-CC-IRDye800CW ( $\left.50 \mathrm{MBq}\right)$ in LNCaP- (A) and PC-3- (B) tumor449 bearing BALB/c nu/nu mice (right flank) 120 min p.i. obtained from small animal PET imaging $(n=1)$. Corresponding 450 time activity curves are shown in (C) for background organs and (D) for tumor and muscle. SUV=standardized uptake 451 value. 


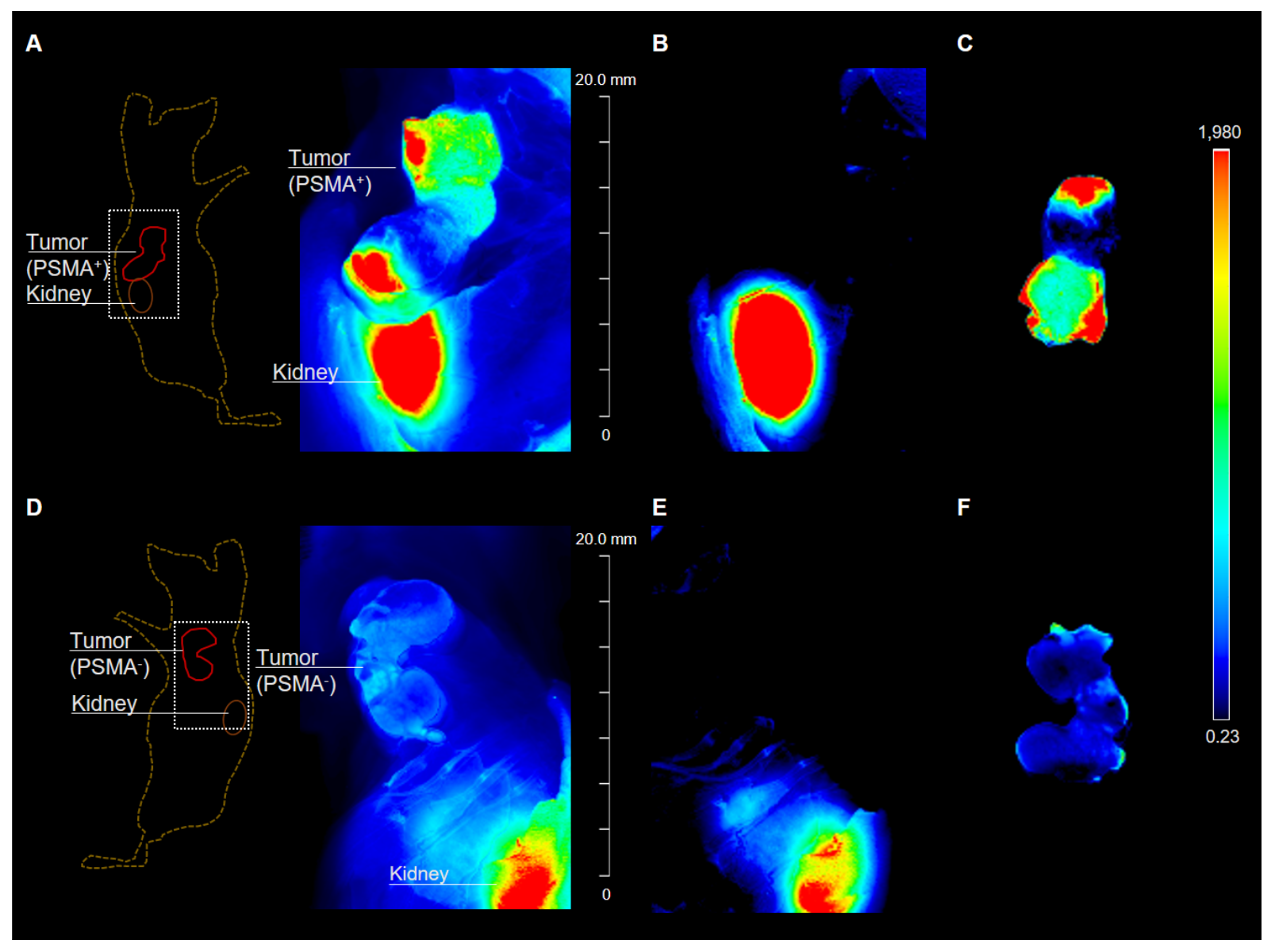

Figure 3. Optical imaging of tumor dissection. Optical imaging was performed after injection of $0.5 \mathrm{nmol}{ }^{68} \mathrm{Ga}-$ labeled Glu-urea-Lys-(HE) 3 -HBED-CC-IRDye800CW in LNCaP (A)-(C) and PC-3 (D)-(F) tumor-bearing BALB/c nu/nu mice $(n=1)$. Mice were sacrificed $2 \mathrm{~h}$ p.i. after PET imaging and fluorescence detected with the Odyssey CLx system (excitation wavelength $800 \mathrm{~nm}$ ). Fluorescence intensity is presented in heat map colouring. Skin was removed covering the subcutaneous xenograft tumors and imaging was performed to locate the tumor (A)/(D). Tumor tissue was resected and another scan performed to ensure complete tumor tissue removal $(B) /(E)$. Fluorescence signal of the resected tumors is presented in $(C) /(F)$. Tissue lying on the surface of the imaging system during fluorescence detection explains small artefacts in fluorescence images. 
464 Table 1. Cell binding and internalization data of the compounds*. Affinity to PSMA and internalization properties 465 of the compounds were determined in vitro using PSMA+-cells (LNCaP). For all compounds PSMA-specific 466 internalization and a not significantly complexation-dependent high binding affinity to PSMA in the low nanomolar 467 range were detected.

\begin{tabular}{|c|c|c|c|c|}
\hline Compound & $\begin{array}{c}\text { Specifically } \\
\text { cell surface } \\
\text { bound } \\
{\left[\% A R / 10^{5}\right.} \\
\text { cells }]^{\dagger}\end{array}$ & $\begin{array}{c}\text { Specifically } \\
\text { internalized } \\
{ }^{\left[\% A R / 10^{5}\right.} \\
{\text { cells }]^{\dagger}}^{\dagger}\end{array}$ & $\mathrm{IC}_{50}[\mathrm{nM}]^{\ddagger}$ & $\begin{array}{c}\mathrm{IC}_{50}[\mathrm{nM}]^{\ddagger} \\
{ }^{69 / 71} \mathrm{Ga}- \\
\text { labeled } \\
\text { compounds }\end{array}$ \\
\hline Glu-urea-Lys-(HE)1-HBED-CC-IRDye800CW & $3.00 \pm 1.42$ & $3.27 \pm 1.83$ & $28.41 \pm 14.39$ & $35.57 \pm 22.83$ \\
\hline Glu-urea-Lys-(WE)1-HBED-CC-IRDye800CW & $6.47 \pm 3.69$ & $13.77 \pm 8.50$ & $43.70 \pm 14.12$ & $56.80 \pm 17.10$ \\
\hline Glu-urea-Lys-(HE) 3 -HBED-CC-IRDye800CW & $8.30 \pm 3.93$ & $6.45 \pm 3.36$ & $51.39 \pm 11.84$ & $69.98 \pm 22.88$ \\
\hline Glu-urea-Lys-HBED-CC-(HE) ${ }_{3}$-IRDye800CW & $6.26 \pm 1.49$ & $3.70 \pm 0.51$ & $41.74 \pm 18.26$ & $52.21 \pm 7.33$ \\
\hline
\end{tabular}

468 * Data are expressed as mean \pm SD $(n=3),+{ }^{68}$ Ga-labeled compounds. Specific cell uptake was determined by 469 blockage using $500 \mu \mathrm{M}$ 2-PMPA. Values are expressed as \% of applied radioactivity (AR) bound to $10^{5}$ cells. $470 \quad \mp$ radioligand: ${ }^{68} \mathrm{Ga}-\mathrm{PSMA}-10$ (Kd: $3.8 \pm 1.8 \mathrm{nM}(25)$, Cradioligand: $\left.0.75 \mathrm{nM}\right)$ 


\section{GRAPHICAL ABSTRACT}
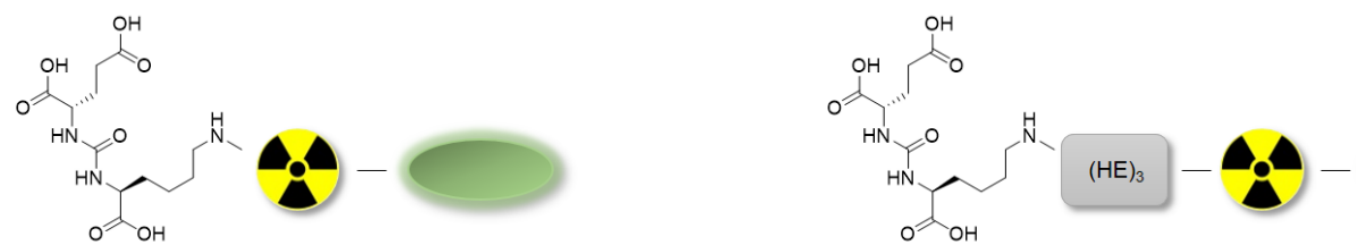

PSMA binding motif Chelator Fluorescent dye

PSMA binding motif Linker Chelator Fluorescent dye

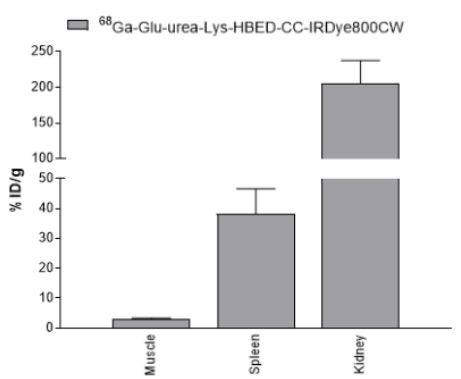

$\stackrel{\text { Rational linker design }}{\longrightarrow}$

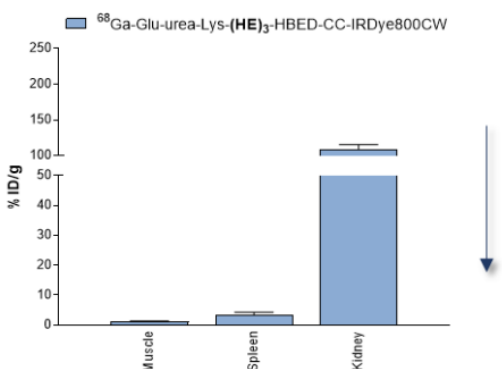

472

Enhanced pharmacokinetic profile 


\section{Supplementary Information}

\section{Compound Synthesis and Characterization}

\section{Supplementary Figures}

Supplemental Figure 1. Synthesis of Glu-urea-Lys-(HE)1-HBED-CC-IRDye800CW, Glu-urea-Lys-(HE)3IRDye800CW and Glu-urea-Lys-(WE)1-HBED-CC-IRDye800CW.

HBED-CC-

Supplemental Figure 2. Synthesis of Glu-urea-Lys-HBED-CC-(HE) $)_{3}-\mathrm{IRDye} 800 \mathrm{CW}$.

Supplemental Figure 3. Analytical HPLC of the compounds.

Supplemental Figure 5. Exemplary analytical radio-HPLC of ${ }^{68} \mathrm{Ga}-\mathrm{Glu}$-urea-Lys-(HE) $)_{3}-$

Supplemental Figure 6. HPLC of co-injected ${ }^{68} \mathrm{Ga}$-Glu-urea-Lys-(HE) $)_{3}$ HBED-CC-IRDye800CW and

Glu-urea-Lys-(HE) $)_{3}$-HBED-CC-IRDye800CW.

Supplemental Figure 7. Reversed-phase thin layer chromatography of ${ }^{68} \mathrm{Ga}$-labeled compounds.

Supplemental Figure 8. Determination of binding affinity to PSMA.

.............................................13

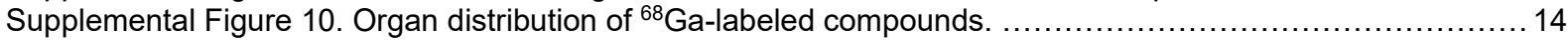

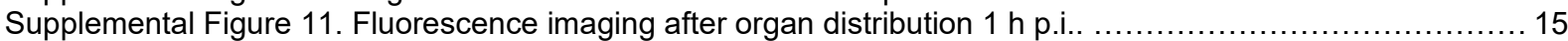

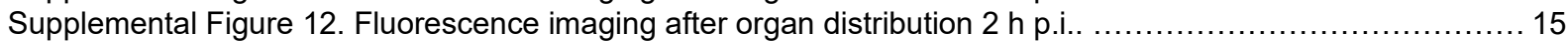

\section{Supplementary Tables}

Supplemental Table 1. Analytical data of the compounds. 16

Supplemental Table 2. Chemical yields of the synthesis intermediates.

Supplemental Table 3. Organ distribution of $0.06 \mathrm{nmol}{ }^{68} \mathrm{Ga}$-labeled compounds in LNCaP-tumor bearing BALB/c nu/nu mice $1 \mathrm{~h}$ p.i..

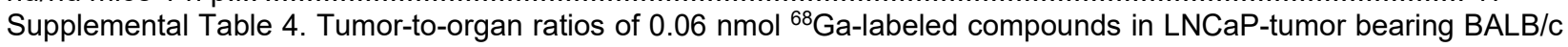
nu/nu mice $1 \mathrm{~h}$ p.i...

Supplemental Table 5. Organ distribution of $0.06 \mathrm{nmol}{ }^{68} \mathrm{Ga}-$ labeled Glu-urea-Lys-(HE)3-HBED-CC-IRDye800CW in $\mathrm{PC}-3$-tumor bearing BALB/c nu/nu mice $1 \mathrm{~h}$ p.i...

Supplemental Table 6. Organ distribution of $0.06 \mathrm{nmol}{ }^{68} \mathrm{Ga}-$ labeled Glu-urea-Lys-(HE) $3-H B E D-C C-I R D y e 800 C W$ in LNCaP-tumor bearing BALB/c nu/nu mice $2 \mathrm{~h}$ p.i. 18

Supplemental Table 7. Organ distribution of $0.5 \mathrm{nmol}{ }^{68} \mathrm{Ga}$-labeled Glu-urea-Lys-(HE) 3 -HBED-CC-IRDye800CW in LNCaP-tumor bearing BALB/c nu/nu mice $1 \mathrm{~h}$ p.i.....

Supplemental Table 8. Organ distribution of $0.5 \mathrm{nmol}{ }^{68} \mathrm{Ga}-$ labeled Glu-urea-Lys-(HE) 3 -HBED-CC-IRDye800CW in $\mathrm{LNCaP}$-tumor bearing BALB/c nu/nu mice $2 \mathrm{~h}$ p.i. 


\section{Compound Synthesis and Characterization}

All commercially available chemicals were of analytical grade and used without further purification. Preparative HPLC was performed with the system LaPrep P110 (VWR) supplied with a variable UV detector (P314, VWR) and a NUCLEODUR® Sphinx RP column (VP250/21, $5 \mu \mathrm{m} 250 \times 21 \mathrm{~mm}$; Macherey-Nagel). The system Agilent 1100 series (Agilent Technologies) was used for semipreparative and analytical HPLC runs thereby analyzing the compounds with reversed-phase high performance liquid chromatography (RP-HPLC; Chromolith RP-18e, $100 \times 10 \mathrm{~mm} /$ Chromolith RP-18e, 100×4.6 mm; Merck). For semipreparative HPLC runs a linear gradient $\left(0.1 \%\right.$ aqueous TFA $(A)$ to $100 \% \mathrm{~B}\left(0.1 \%\right.$ TFA in $\left.\left.\mathrm{CH}_{3} \mathrm{CN}\right)\right)$ in $10 \mathrm{~min}$ at $5 \mathrm{~mL} / \mathrm{min}$ and for analytical HPLC runs a linear gradient $(0.1 \%$ aqueous TFA (A) to $100 \%$ B $(0.1 \%$ TFA in $\left.\mathrm{CH}_{3} \mathrm{CN}\right)$ ) in $10 \mathrm{~min}$ at $2 \mathrm{~mL} / \mathrm{min}$ were selected. UV absorbance was measured at 214 and 254 $\mathrm{nm}$, respectively. For all final products, the chemical purity was greater than $98 \%$, as determined by HPLC. Analytical reversed-phase HPLC of the ${ }^{68}$ Ga-labeled compound Glu-urea-Lys-(HE) $)^{-}$ HBED-CC-IRDye800CW was performed using a EC 250/4.6 NUCLEOSIL 120-5 C 18 column (Machery-Nagel) with a linear gradient $85 \% 0.1 \%$ aqueous TFA (A) to $60 \% \mathrm{~B}(0.1 \%$ TFA in $\mathrm{CH} 3 \mathrm{CN})$ ) in $13 \mathrm{~min}$ at $1.5 \mathrm{~mL} / \mathrm{min}$ or a Chromolith RP-18e $100 \times 4.6 \mathrm{~mm}$ column with a linear gradient $(0.1 \%$ aqueous TFA $(\mathrm{A})$ to $100 \% \mathrm{~B}(0.1 \%$ TFA in $\mathrm{CH} 3 \mathrm{CN}))$ in $5 \mathrm{~min}$ at $4 \mathrm{~mL} / \mathrm{min}$. For mass spectrometry a MALDI-MS (Daltonics Microflex, Bruker Daltonics) and 2,5dihydroxybenzoic acid as a matrix were used. 


\section{Synthesis of Glu-urea-Lys-(HE) ${ }_{1}$-HBED-CC-IRDye800CW, Glu-urea-Lys-(HE) ${ }_{3}$-HBED-CC- IRDye800CW and Glu-urea-Lys-(WE)1-HBED-CC-IRDye800CW}

Synthesis of Glu-urea-Lys-(HE) $)_{1}-\mathrm{CO}\left(\mathrm{CH}_{2}\right)_{4}-\mathrm{N}_{3}$, Glu-urea-Lys-(WE) ${ }_{1}-\mathrm{CO}\left(\mathrm{CH}_{2}\right)_{4}-\mathrm{N}_{3}$ and Glu-ureaLys-(HE) $)_{3}-\mathrm{CO}\left(\mathrm{CH}_{2}\right)_{4}-\mathrm{N}_{3}$

The pharmacophore Glu-urea-Lys was synthesized as described previously (1). Briefly, the isocyanate of the glutamyl moiety was formed using triphosgene in a first step. Resinimmobilized (2-chloro-tritylresin) and $\varepsilon$-allyloxycarbonyl protected lysin was added, the reaction stirred for $16 \mathrm{~h}$ at room temperature and the resin was finally filtered off. By reacting twice with $\mathrm{Pd}\left(\mathrm{PPh}_{3}\right)_{4}$ (0.3 eq.) and morpholine (15 eq.) in dichloromethane under ambient conditions $(1 \mathrm{~h}$, RT, protected from light) the allyloxy-protecting group was cleaved. To build up the different linkers the resin was split and further continued with standard Fmoc solid phase protocols. Depending on the linker sequence the coupling of the amino acids Fmoc-L-His(Trt)-OH, Fmoc-L$\mathrm{Glu}(\mathrm{Ot}-\mathrm{Bu})-\mathrm{OH}$ or Fmoc-L-Trp(Boc)-OH, 4 eq. respectively, and as a final building block 5azidopentanoic acid (4 eq.) was performed using HBTU (4 eq.) and DIPEA (4 eq.) in DMF.

Cleavage of the products from the resin was performed for 3 hours at RT using TFA/TIPS/ $\mathrm{H}_{2} \mathrm{O}$ $(95 / 2.5 / 2.5, v / v / v)$ resulting in the azido-functionalized intermediates, which were purified using RP-HPLC and identified via mass spectrometry.

Synthesis of Glu-urea-Lys-(HE) $1-$ HBED-CC-PEG ${ }_{2}$, Glu-urea-Lys-(WE) $1-$ HBED-CC-PEG 2 and Glu-urea-Lys-(HE) ${ }_{3}$-HBED-CC-PEG 2

As described previously, HBED-CC $(\text { TFP })_{2}$ was synthesized under $\mathrm{Fe}^{3+}$ protection and via the formation of $[\mathrm{Fe}(\mathrm{HBED}-\mathrm{CC})]^{-}$(2). After isolating the bis-TFP ester with preparative HPLC, [Fe (HBED-CC)]TFP 2 and 0.95 eq. of propargylamine were dissolved in DMF in the presence of DIPEA (Supplemental Figure 1). An excess of 2,2'-(ethylenedioxy)bis(ethylamine) (100 $\mu \mathrm{L}$ ) was added after $4 \mathrm{~h}$ at RT and the resulting mixture gently stirred for $16 \mathrm{~h}$ at RT. The alkenylfunctionalized chelator was finally purified using preparative HPLC. Afterwards, $P E G_{2}-$ [Fe(HBED-CC-propargylamine)] (1 eq.) was reacted with the azido-functionalized intermediates 
of the previous step ( 1 eq., respectively) via $\mathrm{CuAAC}, \mathrm{CuSO}_{4}$ (1 eq.), Na-ascorbate (1 eq.), in 3 $\mathrm{mL} \mathrm{THF} / \mathrm{H}_{2} \mathrm{O}(1: 1, \mathrm{v} / \mathrm{v})$ for $16 \mathrm{~h}$ at $\mathrm{RT}$. The Fe-protected products were purified by preparative HPLC and identified with mass spectrometry.

Conjugation of IRDye800CW

IRDye800CW-NHS ester (1 eq.) was conjugated to Glu-urea-Lys-(HE) ${ }_{1}$-[Fe(HBED-CC-PEG $2^{-}$ $\left.\mathrm{NH}_{2}\right)$, Glu-urea-Lys-(WE) 1 -[Fe(HBED-CC-PEG $\left.\left.{ }_{2}-\mathrm{NH}_{2}\right)\right]$ and Glu-urea-Lys-(HE) $)_{3}-[\mathrm{Fe}(\mathrm{HBED}-\mathrm{CC}-$ $\left.\mathrm{PEG}_{2}-\mathrm{NH}_{2}\right)$ ], respectively, in PBS-buffer $(\mathrm{pH} 8.5)$ for $24 \mathrm{~h}$ at $\mathrm{RT}$. The Fe-protected products were isolated via semipreparative HPLC and identified with mass spectrometry. Complexed $\mathrm{Fe}^{3+}$ was removed as described previously (2). Briefly, the Fe-containing products were trapped on a C18 cartridge. Subsequently, the cartridge was flushed with $10 \mathrm{~mL} 1 \mathrm{M} \mathrm{HCl}$ and washed with $5 \mathrm{~mL}$ $\mathrm{H}_{2} \mathrm{O}$. The final products were eluted with $2 \mathrm{~mL} \mathrm{H}_{2} \mathrm{O} / \mathrm{CH}_{3} \mathrm{CN}(3: 1)$ and evaporated to dryness. 

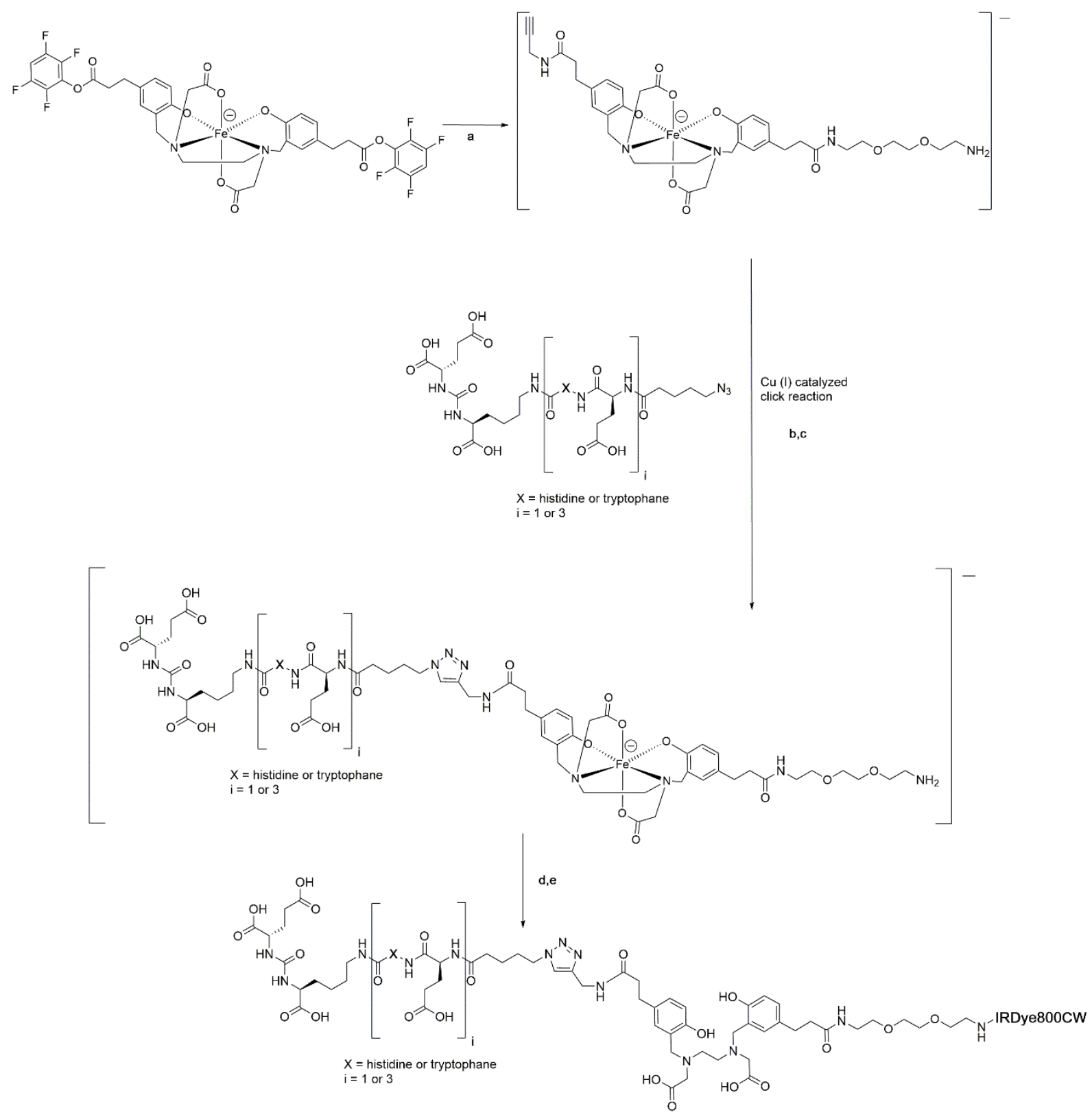

Supplemental Figure 1. Synthesis of Glu-urea-Lys-(HE) 1 -HBED-CC-IRDye800CW, Glu-urea-Lys-(HE) $)_{3}$-HBEDCC-IRDye800CW and Glu-urea-Lys-(WE)1-HBED-CC-IRDye800CW. (a) 0.95 eq. 2-propynylamine, DIPEA, 4 h, RT; excess $\left(100 \mu \mathrm{L}\right.$ ) 2,2'-(ethylendioxy)bis(ethylamine), 16 h, RT, (b) 1 eq. CuSO4, (c) 1 eq. Na-ascorbate in THF/ $\mathrm{H}_{2} \mathrm{O}$ (1:1, v/v), RT, 16 h, (d) PBS-buffer (pH 8.5), 1 eq. IRDye800CW-NHS ester RT, 16 h, (e) $1 \mathrm{M} \mathrm{HCl}$. 


\section{Synthesis of Glu-urea-Lys-HBED-CC-(HE) ${ }_{3}$-IRDye800CW}

In a first step $\mathrm{PEG}_{2}-(\mathrm{EH})_{3}-\mathrm{CO}\left(\mathrm{CH}_{2}\right)_{4}-\mathrm{N}_{3}$ was synthesized starting from an OBis(aminoethyl)ethylene glycol trityl resin. Based on a standard Fmoc solid phase protocol, Fmoc-L-Glu(Ot-Bu)-OH and Fmoc-L-His(Trt)-OH (4 eq. each) were activated with HBTU (4 eq.) and DIPEA (4 eq.) and coupled in DMF. The couplings were repeated three times to obtain the $(\mathrm{HE})_{3}$-motif. Finally, 5-azidopentanoic acid (4 eq.) was coupled using HBTU (4 eq.) and DIPEA (4 eq.) in DMF. The azido-functionalized intermediate was cleaved from the resin for 3 hours at RT using TFA/TIPS/ $\mathrm{H}_{2} \mathrm{O}(95 / 2.5 / 2.5, \mathrm{v} / \mathrm{v} / \mathrm{v})$, the product purified with preparative HPLC and identified with mass spectrometry.

Subsequently, bis-TFP activated [Fe(HBED-CC)]- (1) was reacted with tris $(t-B u)$-protected Gluurea-Lys (ABX; 0.9 eq.) in DMF at RT (Supplemental Figure 2). After $4 \mathrm{~h}, 50 \mu \mathrm{L} 2$ propynylamine was added and the mixture stirred for $16 \mathrm{~h}$ at RT. The resulting Glu-ureaLys([Fe(HBED-CC-propargylamin)]) was isolated via preparative HPLC (2). PEG $2_{2}(E H)_{3^{-}}$ $\mathrm{CO}\left(\mathrm{CH}_{2}\right)_{4}-\mathrm{N}_{3}$ (1 eq.) and tris(t-Bu)-protected Glu-urea-Lys([Fe(HBED-CC-propargylamine)]) (1 eq.) were reacted via CuAAC, $\mathrm{CuSO}_{4}$ (1 eq.), Na-ascorbate (1 eq.), in $3 \mathrm{~mL} \mathrm{THF} / \mathrm{H}_{2} \mathrm{O}(1: 1, \mathrm{v} / \mathrm{v})$ for $16 \mathrm{~h}$ at $\mathrm{RT}$. The tris $(t-\mathrm{Bu})$ - and Fe-protected intermediate was separated by preparative HPLC and the tris $(t-\mathrm{Bu})$-protecting groups removed after lyophilisation with $3 \mathrm{~mL}$ TFA for $4 \mathrm{~h}$ at RT (3). In the final step IRDye800CW-NHS ester was conjugated to Glu-urea-Lys([Fe(HBED$\left.\left.\left.\mathrm{CC}-(\mathrm{HE})_{3}-\mathrm{PEG}_{2}-\mathrm{NH}_{2}\right)\right]\right)$ and complexed $\mathrm{Fe}^{3+}$ removed as described in the previous section (4). 


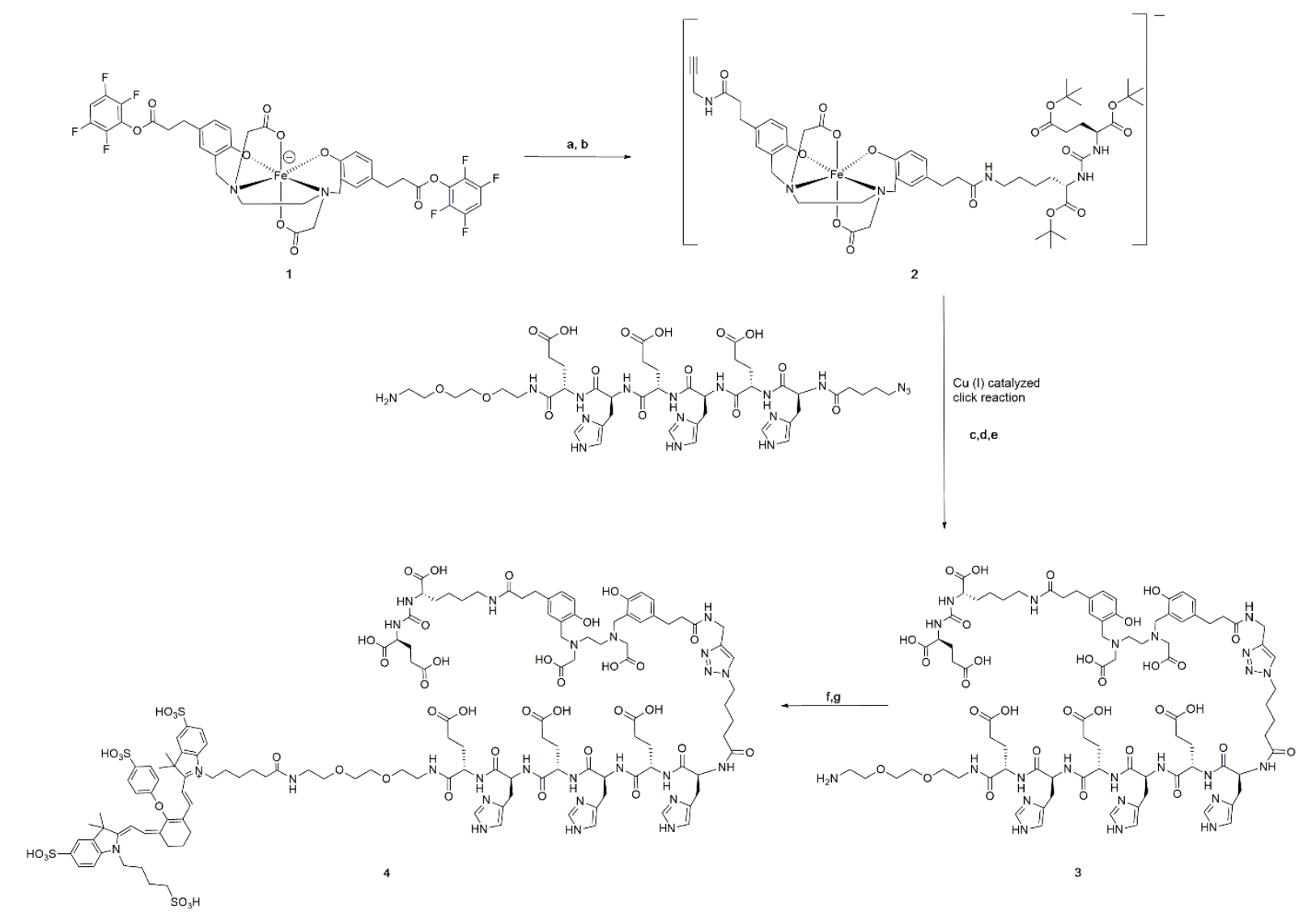

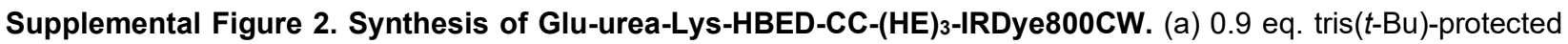
Glu-urea-Lys in DMF, DIPEA, 4 h, RT (b) excess (50 $\mu \mathrm{L})$ 2-propynylamine, $16 \mathrm{~h}, \mathrm{RT}$, (c) 1 eq. CuSO4, d) 1 eq. Naascorbate in THF/H $/ \mathrm{H}_{2} \mathrm{O}(1: 1, \mathrm{v} / \mathrm{v}), \mathrm{RT}, 16 \mathrm{~h}$, (e) TFA, $3 \mathrm{~h}, \mathrm{RT}$, (f) PBS-buffer (pH 8.5), 1 eq. IRDye800CW-NHS ester $\mathrm{RT}, 16 \mathrm{~h},(\mathrm{~g}) 1 \mathrm{M} \mathrm{HCl}$.

\section{Radiolabeling with ${ }^{68} \mathrm{Ga}$ and Determination of Lipophilicity}

The precursor peptides [1 nmol in 2-[4-(2-hydroxyethyl)piperazin-1-yl]ethanesulfonic acid (HEPES) buffer $(580 \mathrm{mg} / \mathrm{ml})$ with $5 \mathrm{mg}$ ascorbic acid, $90 \mu \mathrm{L}]$ were added to $40 \mu \mathrm{L}{ }^{68} \mathrm{Ga}^{3+}$ eluate ( $80-120 \mathrm{MBq})$. The $\mathrm{pH}$ was adjusted to 3.8 using $30 \% \mathrm{NaOH}$ and $10 \% \mathrm{NaOH}$, respectively. The reaction mixture was incubated at $98^{\circ} \mathrm{C}$ for 10 minutes. The respective radiochemical yield was determined by reversed-phase high performance liquid chromatography (RP-HPLC) or reversed-phase thin layer chromatography (RP-TLC, 60 RP-18 $F_{254 \mathrm{~S}}$ ) with $0.1 \mathrm{M}$ sodium citrate [HOC(COONa) $\left.\left(\mathrm{CH}_{2} \mathrm{COONa}\right)_{2} \cdot 2 \mathrm{H}_{2} \mathrm{O}\right]$ as mobile phase. The 2-phase system n-octanol and PBS was used for the determination of lipophilicity of the ${ }^{68} \mathrm{Ga}-$ labeled compounds. 


\section{In Vitro Serum Stability}

${ }^{68}$ Ga-labeled Glu-urea-Lys-(HE) $)_{3}$-HBED-CC-IRDye800CW $(10 \mu \mathrm{L})$ was incubated in $100 \mu \mathrm{L}$ human or mouse plasma $(100 \mu \mathrm{L})$ at $37^{\circ} \mathrm{C}$. At different time points of incubation $(\mathrm{t}=1 \mathrm{~h}, 2 \mathrm{~h})$, aliquots of $10 \mu \mathrm{L}$ were taken and plasma proteins precipitated in $30 \mu \mathrm{L}$ acetonitrile. Samples were centrifuged for $5 \mathrm{~min}$ at $13000 \mathrm{rpm}$ and the supernatants analysed by analytical RP-HPLC.

\section{Preclinical Proof-of-Concept Study (PET and Optical Imaging)}

The PET imaging study was performed with an Inveon $\mu$-PET scanner (Siemens) with a dynamic scan for $60 \mathrm{~min}$ and a static scan $(10 \mathrm{~min})$ at $2 \mathrm{~h}$ p.i.. Transmission scans $(10 \mathrm{~min})$ were performed before the dynamic and static image acquisition, respectively. The images were reconstructed with the Inveon Acquisition Workspace Software (Siemens) and OSEM 3D SPMAP algorithm (16 subsets, 4 iterations, MAP Iteration: 18) using a 28 frame protocol $(2 \times 15 s, 8 \times 30 s, 5 \times 60 s, 5 \times 120 s, 8 \times 300 s)$ for the dynamic scan and one frame for the static scan. Median root was applied prior correction and images were converted to SUV images.

To identify the subcutaneous tumor by fluorescence, optical imaging was performed after PET imaging. Mice were sacrificed and the skin was removed covering the subcutaneous xenograft tumors. Subsequently, the Odyssey CLx system (LI-COR Biosciences, excitation wavelength $800 \mathrm{~nm}$ ) was used for locating the tumor by fluorescence. Tumor tissue was resected and another scan performed with the Odyssey CLx system to ensure complete tumor tissue removal. 


\section{Supplementary Figures}

A

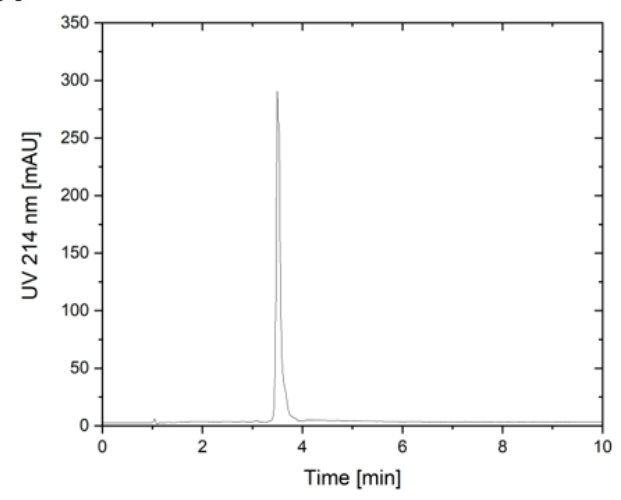

C

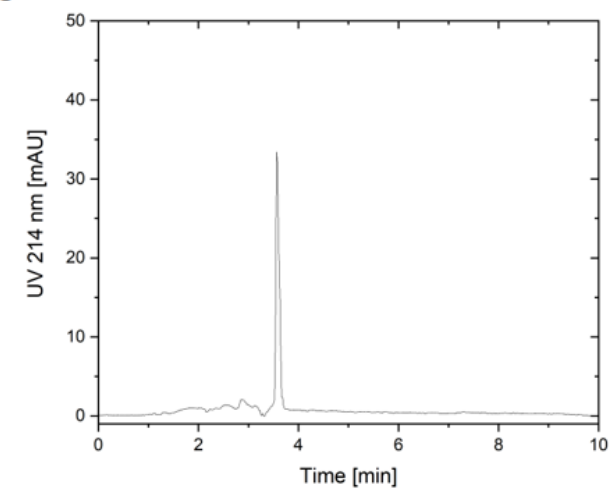

B
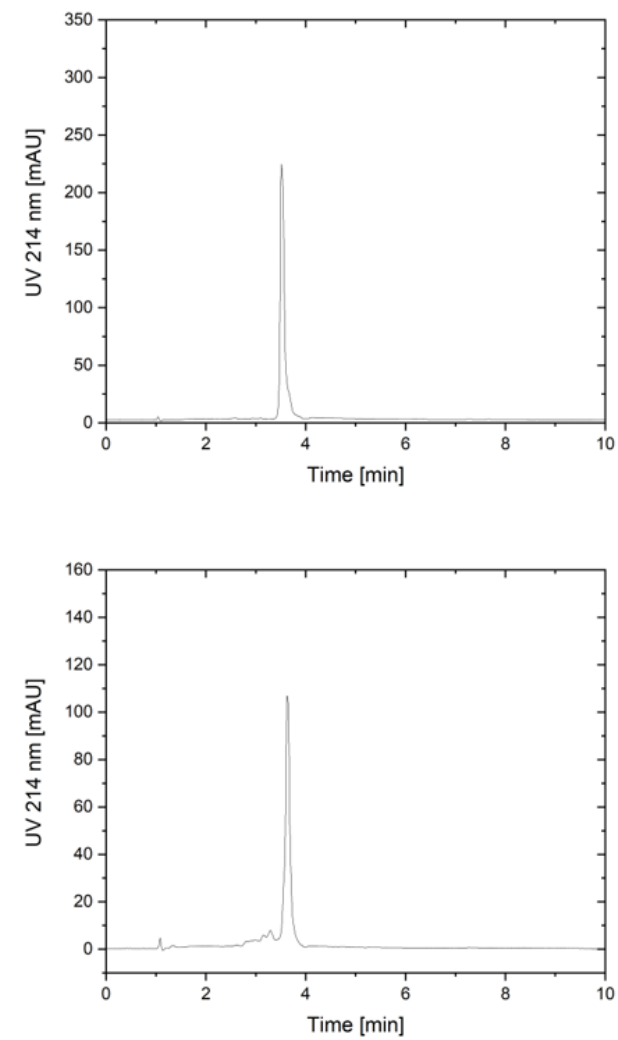

Supplemental Figure 3. Analytical HPLC of the compounds. Analytical reversed-phase HPLC of the final compounds was performed using a Chromolith RP-18e $100 \times 4.6 \mathrm{~mm}$ column with a linear gradient $\left(0.1 \% \mathrm{TFA}\right.$ in $\mathrm{H}_{2} \mathrm{O}$ (A) to $100 \% \mathrm{~B}\left(0.1 \% \mathrm{TFA}\right.$ in $\left.\left.\mathrm{CH}_{3} \mathrm{CN}\right)\right)$ in $10 \mathrm{~min}$ at $2 \mathrm{~mL} / \mathrm{min}$. UV trace at $214 \mathrm{~nm}$ is shown. (A) Glu-urea-Lys-(HE) 1 HBED-CC-IRDye800CW, (B) Glu-urea-Lys-(WE)1-HBED-CC-IRDye800CW, (C) Glu-urea-Lys-(HE) 3 -HBED-CCIRDye800CW and (D) Glu-urea-Lys-HBED-CC-(HE)3-IRDye800CW. Original data was plotted using OriginPro 2020 software. 
A

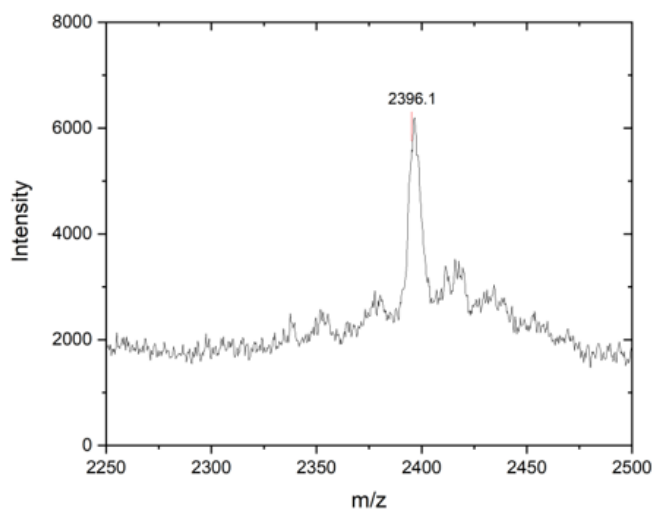

C

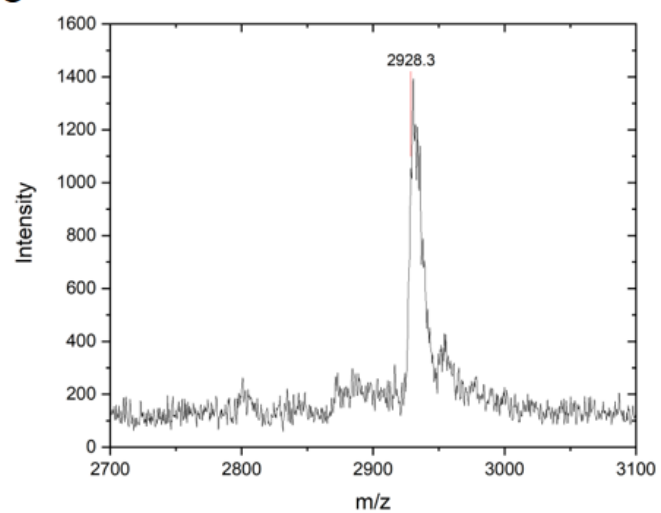

B

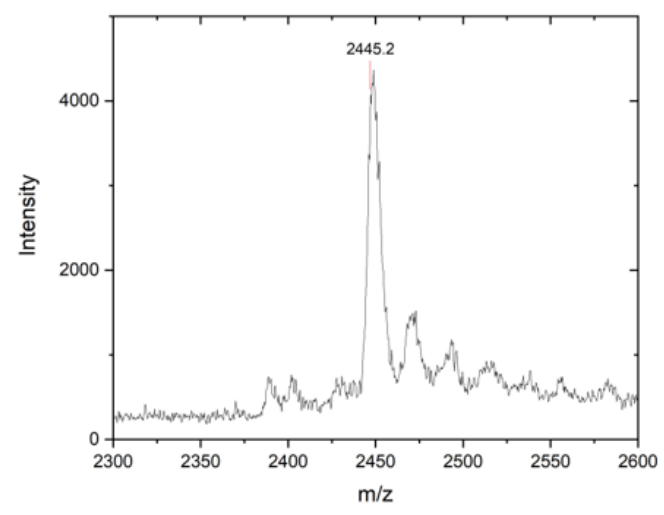

D

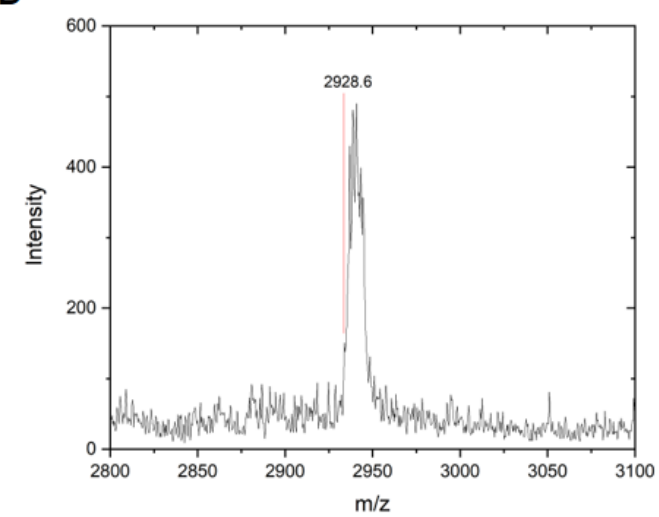

Supplemental Figure 4. MALDI spectra of the compounds. Mass spectrometry was performed with a MALDI-MS (Daltonics Microflex, Bruker Daltonics) and 2,5-dihydroxybenzoic acid as a matrix. (A) Glu-urea-Lys-(HE)1-HBED-CCIRDye800CW, (B) Glu-urea-Lys-(WE) 1-HBED-CC-IRDye800CW, (C) Glu-urea-Lys-(HE) 3 -HBED-CC-IRDye800CW and (D) Glu-urea-Lys-HBED-CC-(HE)3-IRDye800CW. Original data was plotted using OriginPro 2020 software. 


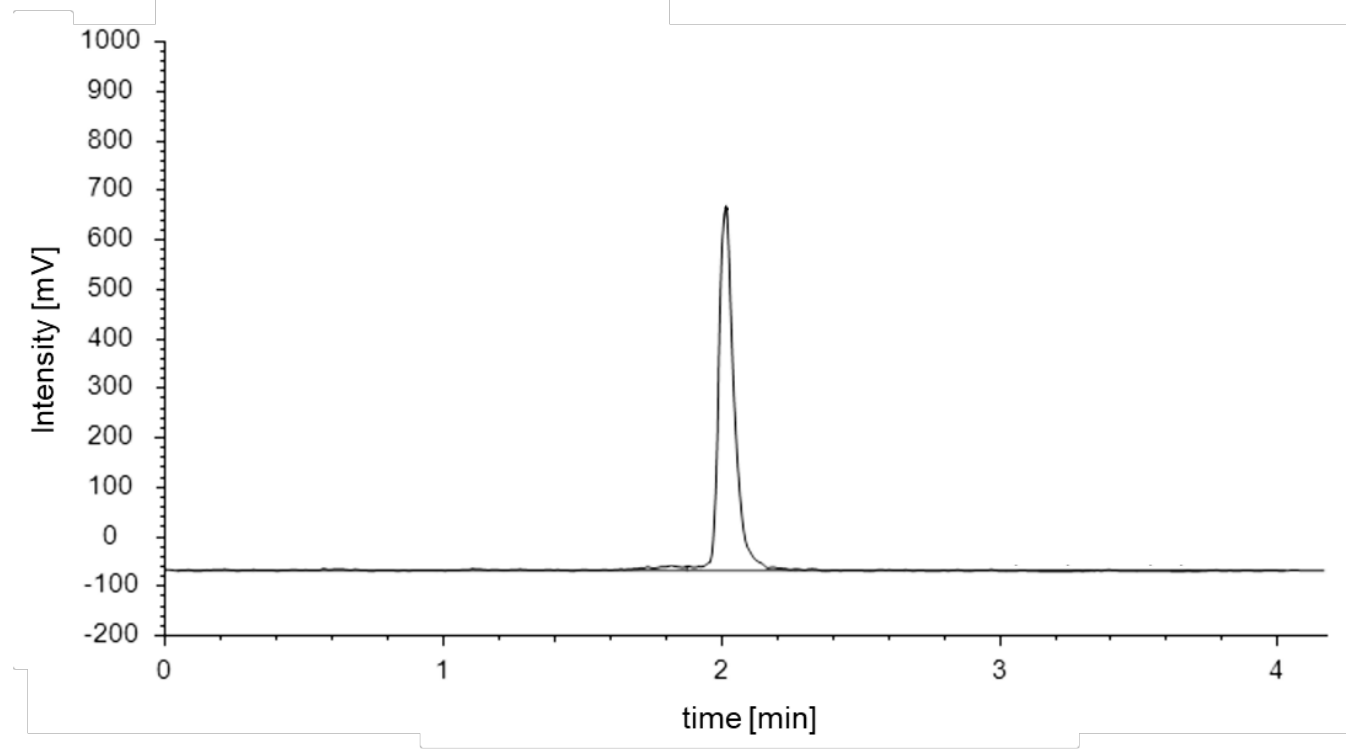

Supplemental Figure 5. Exemplary analytical radio-HPLC of ${ }^{68} \mathrm{Ga}-\mathrm{Glu}-\mathrm{urea}-\mathrm{Lys}-(\mathrm{HE}){ }_{3}-\mathrm{HBED}-\mathrm{CC}-\mathrm{IRDye} 800 \mathrm{CW}$. Analytical reversed-phase HPLC of the ${ }^{68} \mathrm{Ga}$-labeled compound was performed using a Chromolith RP-18e $100 \times 4.6$ $\mathrm{mm}$ column with a linear gradient $\left(0.1 \%\right.$ aqueous TFA $(\mathrm{A})$ to $100 \% \mathrm{~B}\left(0.1 \%\right.$ TFA in $\left.\left.\mathrm{CH}_{3} \mathrm{CN}\right)\right)$ in $5 \mathrm{~min}$ at $4 \mathrm{~mL} / \mathrm{min}$. For radiodetection the system was equipped with a $\gamma$-detector.

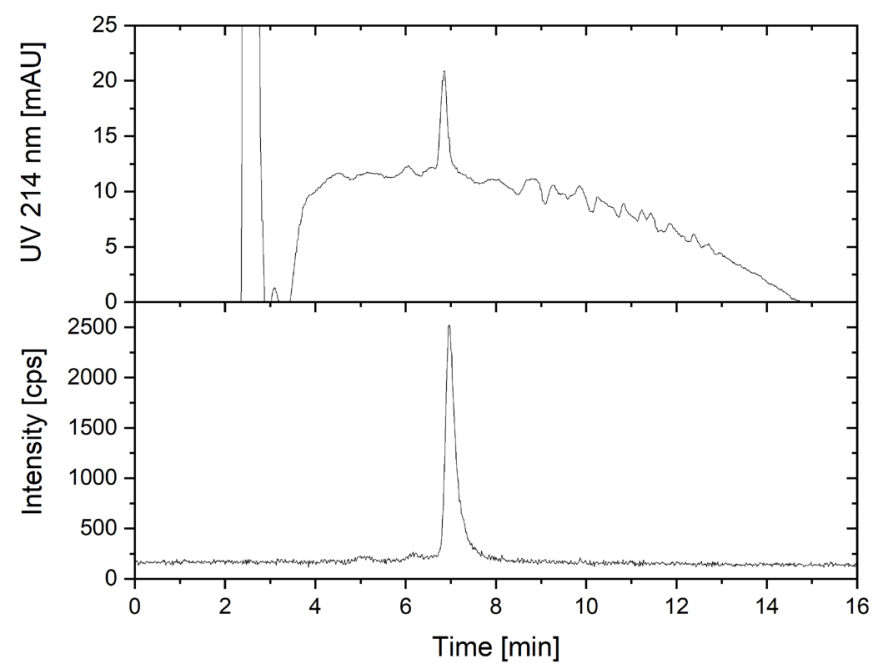

Supplemental Figure 6. HPLC of co-injected ${ }^{68}$ Ga-Glu-urea-Lys-(HE)3-HBED-CC-IRDye800CW and Glu-ureaLys-(HE)3-HBED-CC-IRDye800CW. Analytical reversed-phase HPLC of the ${ }^{68} \mathrm{Ga}-$ labeled compound co-injected with the non-radiolabeled precursor Glu-urea-Lys-(HE) 3 -HBED-CC-IRDye800CW was performed using a EC 250/4.6 NUCLEOSIL 120-5 C 18 column (Machery-Nagel) with a linear gradient $85 \% 0.1 \%$ aqueous TFA (A) to $60 \%$ B (0.1\% TFA in $\mathrm{CH}_{3} \mathrm{CN}$ )) in $13 \mathrm{~min}$ at $1.5 \mathrm{~mL} / \mathrm{min}$. UV was measured at $214 \mathrm{~nm}$ and for radiodetection the system was equipped with a $\mathrm{y}$-detector. Original data was plotted using OriginPro 2020 software. 
A

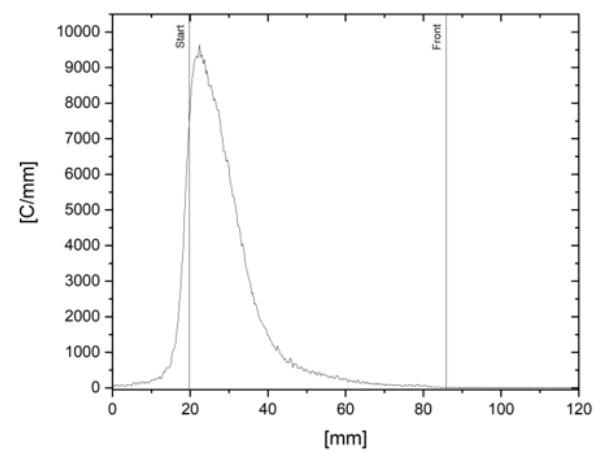

C

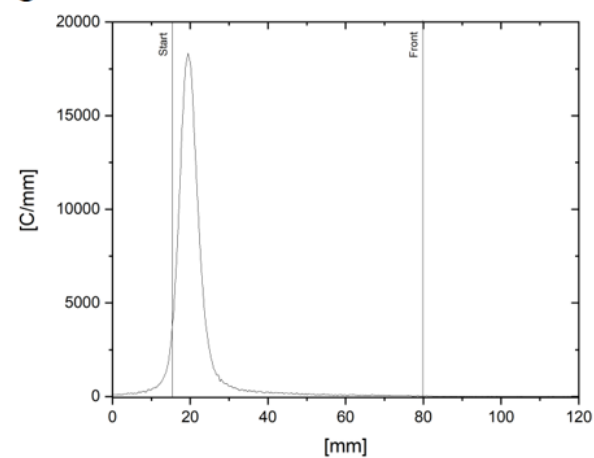

E

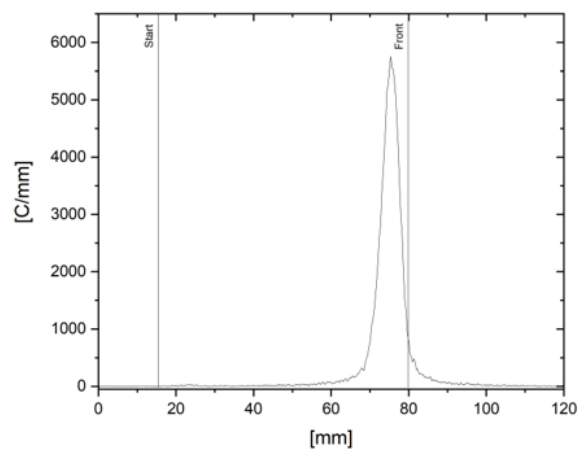

B

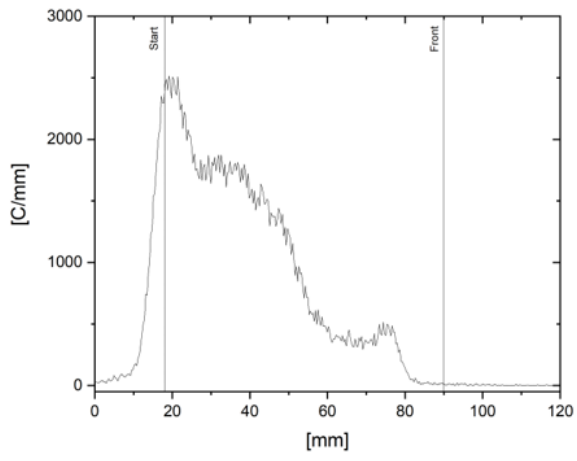

D

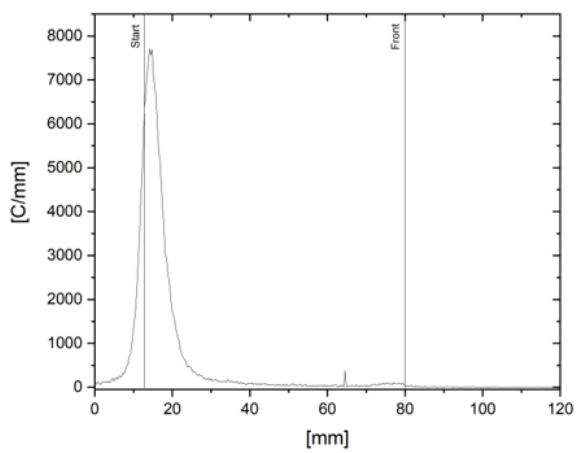

Supplemental Figure 7. Reversed-phase thin layer chromatography of ${ }^{68}$ Ga-labeled compounds. RP-TLC (60 RP-18 $\mathrm{F}_{254 \mathrm{~S}}$ ) was performed with $0.1 \mathrm{M}$ sodium citrate as mobile phase. (A) Glu-urea-Lys-(HE) 1 -HBED-CCIRDye800CW, (B) Glu-urea-Lys-(WE)1-HBED-CC-IRDye800CW, (C) Glu-urea-Lys-(HE) 3 -HBED-CC-IRDye800CW, (D) Glu-urea-Lys-HBED-CC-(HE) ${ }_{3}-\mathrm{IRDye} 800 \mathrm{CW}$ and $(\mathrm{E}){ }^{68} \mathrm{GaCl}_{3}$ as a control. Original data was plotted using OriginPro 2020 software. 
A

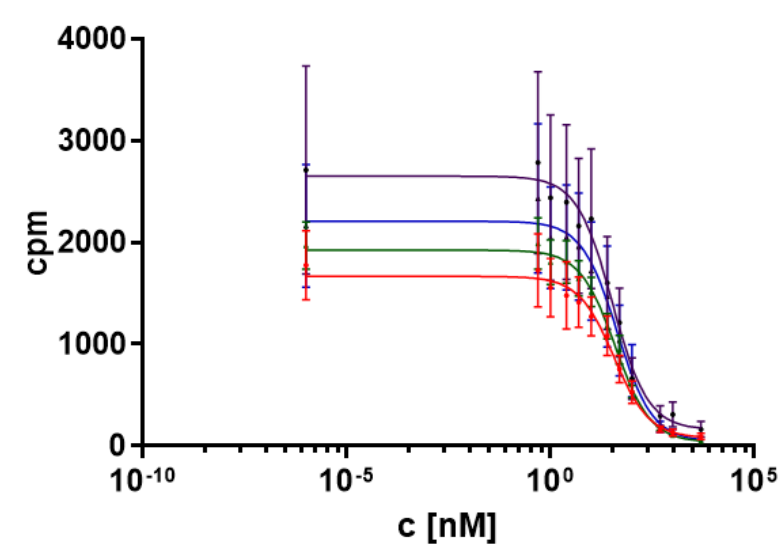

B

- Glu-urea-Lys-(HE) ${ }_{1}$-HBED-CC-IRDye800CW

- Glu-urea-Lys-(WE) $)_{1}$-HBED-CC-IRDye800CW

- Glu-urea-Lys-(HE) $)_{3}$-HBED-CC-IRDye800CW

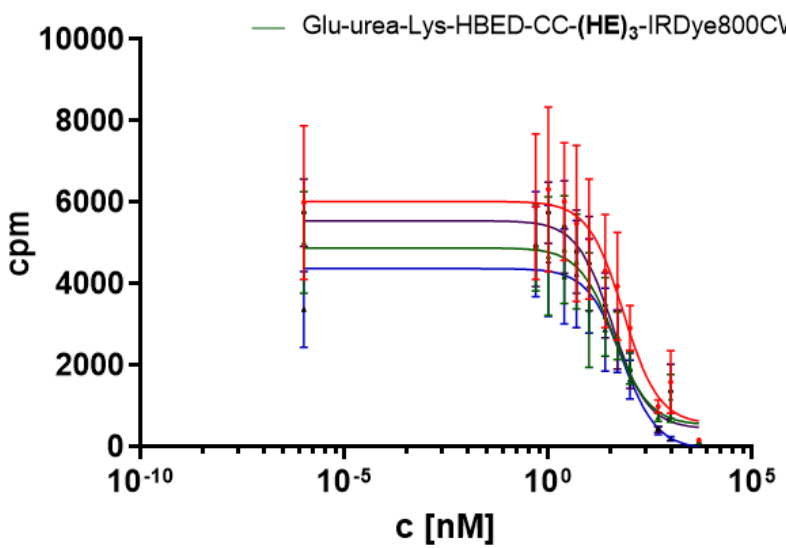

Supplemental Figure 8. Determination of binding affinity to PSMA. Binding affinity was determined on PSMA ${ }^{+}$ LNCaP-cells with ${ }^{68} \mathrm{Ga}-\mathrm{PSMA}-10\left(\mathrm{~K}_{\mathrm{d}}: 3.8 \pm 1.8 \mathrm{nM}(1)\right.$, Cradioligand: $\left.0.75 \mathrm{nM}\right)$ as radioligand. $\mathrm{IC}_{50}$ curves of $(\mathrm{A})$ free ligands and (B) ${ }^{69 / 71} \mathrm{Ga}$-labeled ligands were plotted with GraphPad Software. The $50 \%$ inhibitory concentration (IC50) values were calculated by fitting the data using a nonlinear regression algorithm (GraphPad Software).

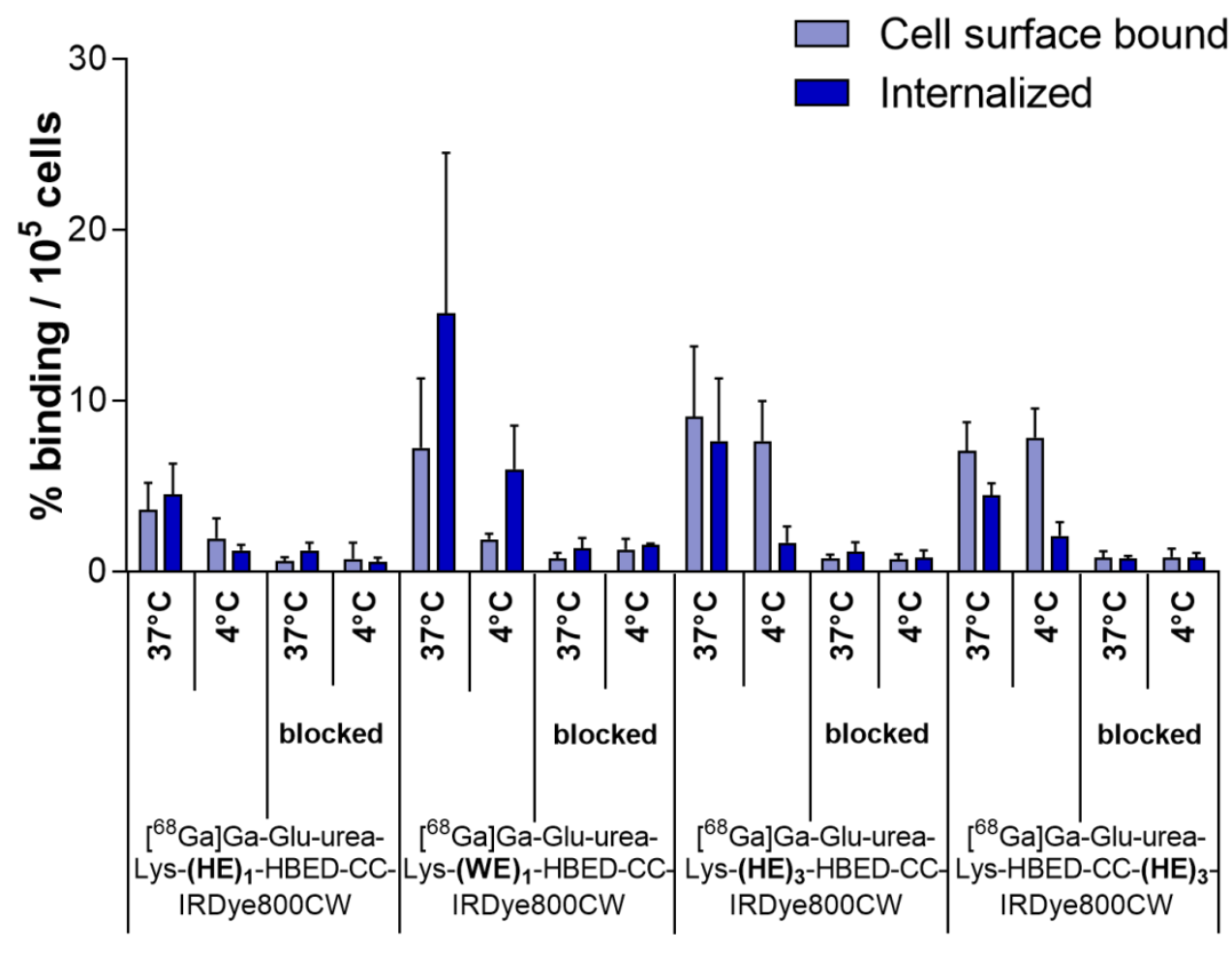

Supplemental Figure 9. Cell surface binding and internalization of ${ }^{68} \mathrm{Ga}$-labeled compounds. Specificity of internalization was determined by blocking with 2-PMPA $(500 \mu \mathrm{M})$ or incubating on ice at $4^{\circ} \mathrm{C}$. Data are expressed in $\%$ binding $/ 10^{5}$ cells and represent mean \pm standard deviation $(n=3)$. 


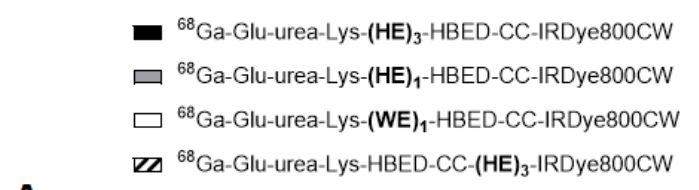

A

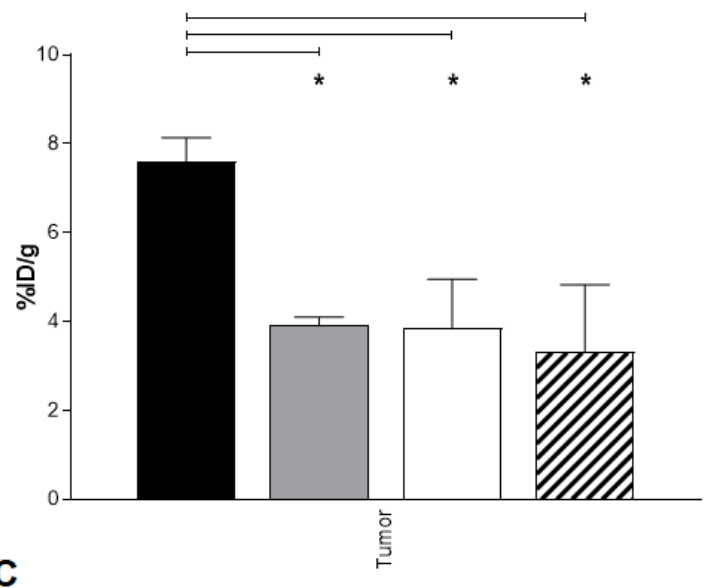

C

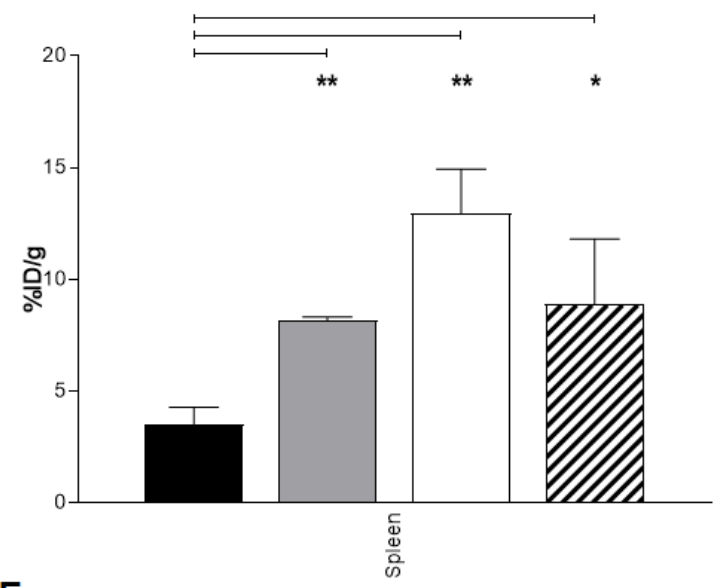

$\mathbf{E}$

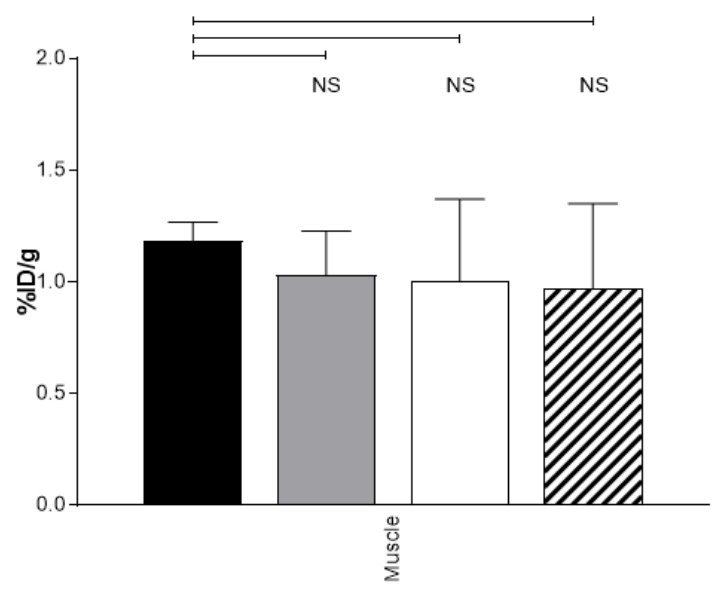

B
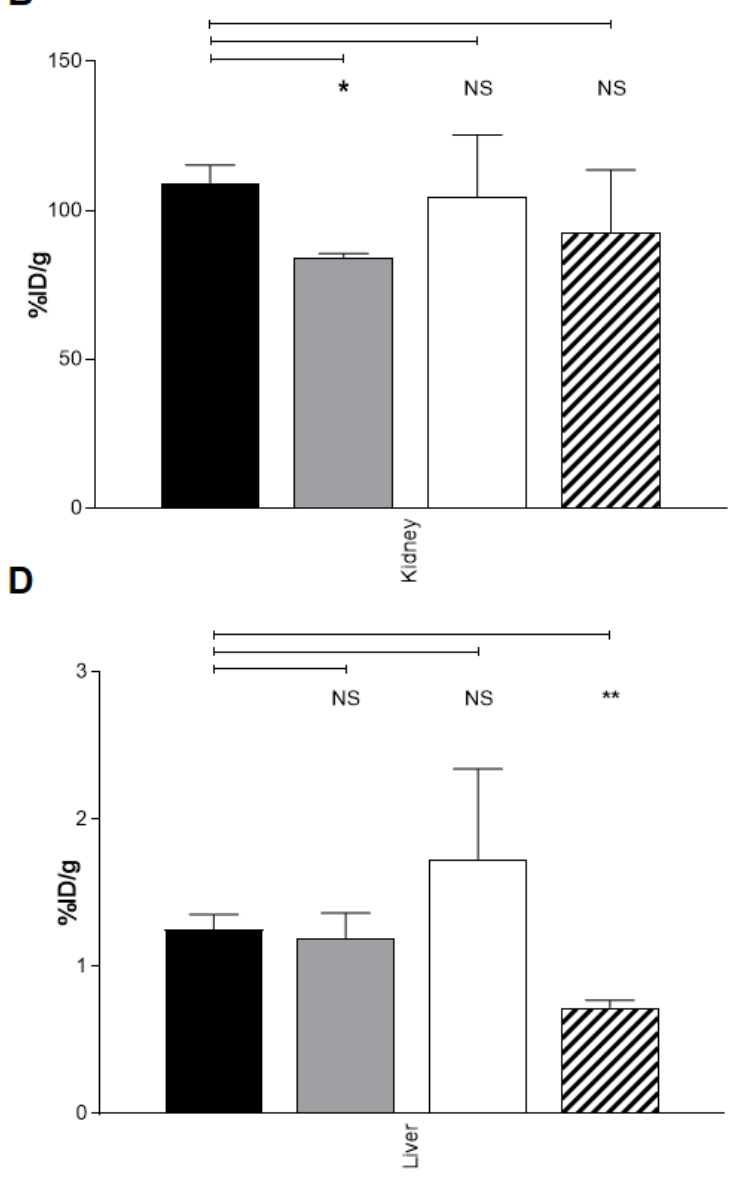

$\mathbf{F}$

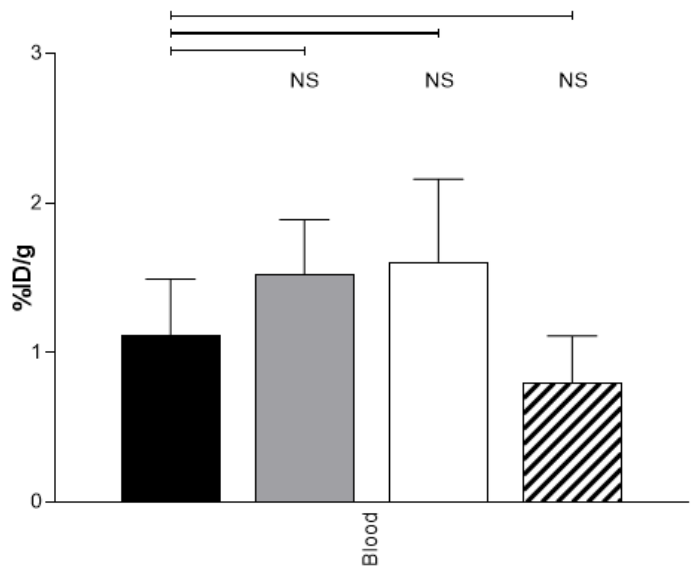

Supplemental Figure 10. Organ distribution of ${ }^{68} \mathrm{Ga}$-labeled compounds. Organ distribution of $60 \mathrm{pmol}{ }^{68} \mathrm{Ga}-$ labeled compounds at $1 \mathrm{~h}$ p.i. with uptake in (A) PSMA ${ }^{+}$-tumor (LNCaP), (B) kidney, (C) spleen, (D) liver, (E) muscle and $(F)$ blood. Data are expressed as mean \% ID/g tissue \pm SD $(n=3)$. Significant differences are presented with asterisks above the bars that are being compared (NS: not significant, ${ }^{*} p<0.05,{ }^{* *} p<0.01$ ). 


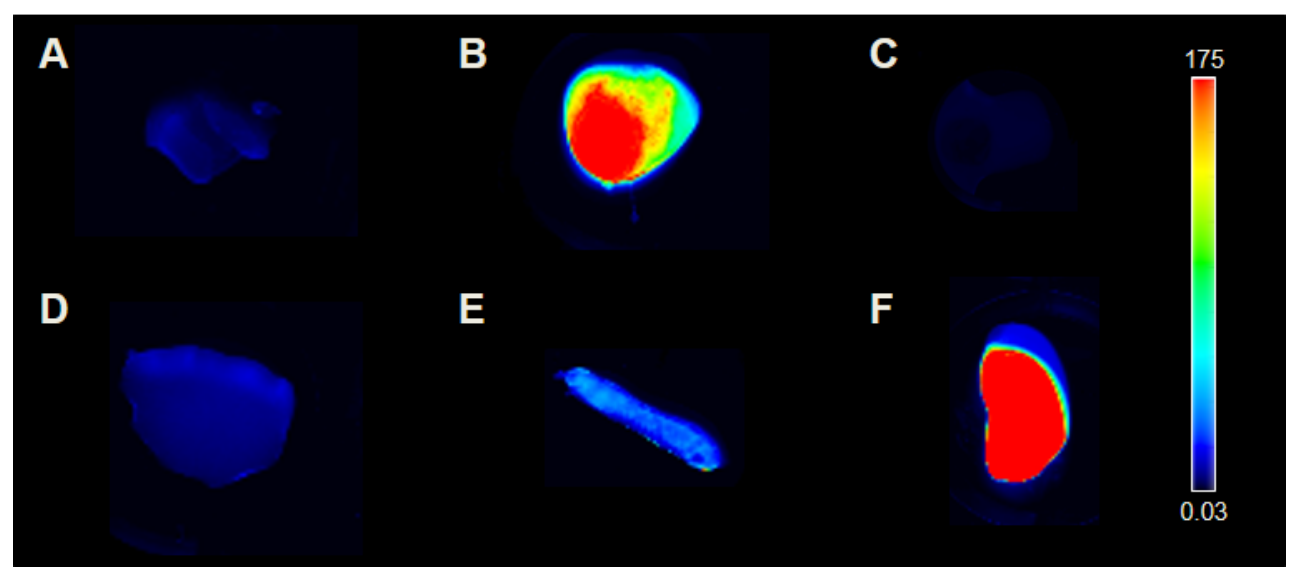

Supplemental Figure 11. Fluorescence imaging after organ distribution $1 \mathbf{h}$ p.i.. Fluorescence imaging of organs dissected after injection of $0.5 \mathrm{nmol}{ }^{68} \mathrm{Ga}$-labeled Glu-urea-Lys-(HE) 3 -HBED-CC-IRDye800CW in LNCaP tumorbearing BALB/c nu/nu mice $1 \mathrm{~h}$ p.i. ( $n=3$, one representative image out of three is shown) acquired with the Odyssey CLx system (excitation wavelength $800 \mathrm{~nm}$ ). Fluorescence intensity is presented in heat map colouring. (A) muscle, (B) PSMA+-tumor (LNCaP), (C) blood (D) liver, (E) spleen, (F) kidney.

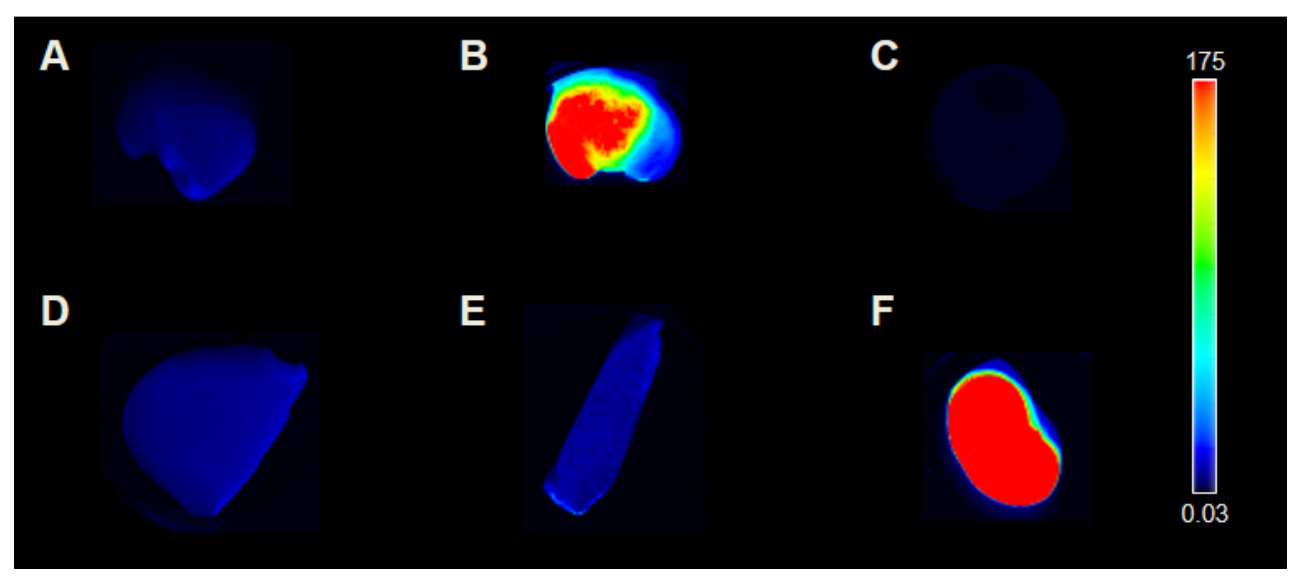

Supplemental Figure 12. Fluorescence imaging after organ distribution $\mathbf{2}$ h p.i.. Fluorescence imaging of organs dissected after injection of $0.5 \mathrm{nmol}{ }^{68} \mathrm{Ga}$-labeled Glu-urea-Lys-(HE) 3 -HBED-CC-IRDye800CW in LNCaP tumorbearing BALB/c nu/nu mice $2 \mathrm{~h} \mathrm{p.i.} \mathrm{(} n=3$, one representative image out of three is shown) acquired with the Odyssey CLx system (excitation wavelength $800 \mathrm{~nm}$ ). Fluorescence intensity is presented in heat map colouring. (A) muscle, (B) PSMA+-tumor (LNCaP), (C) blood (D) liver, (E) spleen, (F) kidney. 


\section{Supplementary Tables}

Supplemental Table 1. Analytical data of the compounds.

\begin{tabular}{|c|c|c|c|c|c|c|}
\hline Compound & Sum formula & $\begin{array}{c}\mathrm{m} / \mathrm{z} \\
\text { exact mass (calc.) }\end{array}$ & $\mathrm{m} / \mathrm{z}^{*}$ & $\begin{array}{l}\text { Chemical } \\
\text { yield [\%] }]^{\dagger}\end{array}$ & $\begin{array}{c}\text { Lipophilicity } \\
\log D_{\mathrm{pH}} 7.4 \\
\text { n-octanol/PBS }\end{array}$ & $\begin{array}{l}* \\
\text { Mass } \\
\text { spect }\end{array}$ \\
\hline $\begin{array}{l}\text { Glu-urea-Lys-(HE)1- } \\
\text { HBED-CC-IRDye800CW }\end{array}$ & $\mathrm{C}_{1090} \mathrm{H}_{144} \mathrm{~N}_{17} \mathrm{O}_{36} \mathrm{~S}_{4}{ }^{+}$ & 2394.9 & 2396.1 & 34 & $-3.15 \pm 0.08$ & $\begin{array}{l}\text { try of } \\
\text { non- }\end{array}$ \\
\hline $\begin{array}{l}\text { Glu-urea-Lys-(WE)1- } \\
\text { HBED-CC-IRDye800CW }\end{array}$ & $\mathrm{C}_{114} \mathrm{H}_{147} \mathrm{~N}_{16} \mathrm{O}_{36} \mathrm{~S}_{4}{ }^{+}$ & 2443.9 & 2445.2 & 12 & $-3.03 \pm 0.05$ & ed \\
\hline $\begin{array}{l}\text { Glu-urea-Lys-(HE) }{ }_{3-}^{-} \\
\text {HBED-CC-IRDye800CW }\end{array}$ & $\mathrm{C}_{131} \mathrm{H}_{172} \mathrm{~N}_{25} \mathrm{O}_{44} \mathrm{~S}_{4}{ }^{+}$ & 2927.1 & 2928.3 & 38 & $-1.65 \pm 0.40$ & dete \\
\hline $\begin{array}{l}\text { Glu-urea-Lys-HBED-CC- } \\
\text { (HE) })_{3}-\text { IRDye800CW }\end{array}$ & $\mathrm{C}_{131} \mathrm{H}_{172} \mathrm{~N}_{25} \mathrm{O}_{44} \mathrm{~S}_{4}{ }^{+}$ & 2927.1 & 2928.6 & 17 & $-3.22 \pm 0.08$ & $\begin{array}{l}{[\mathrm{M}+} \\
\mathrm{H}]^{+},+ \\
\mathrm{Che}\end{array}$ \\
\hline
\end{tabular}

mical yields refer to the fluorescent dye conjugation, ${ }^{\ddagger}$ Lipophilicity of ${ }^{68} \mathrm{Ga}$-labeled ligand.

Supplemental Table 2. Chemical yields of the synthesis intermediates.

\begin{tabular}{|c|c|}
\hline Intermediate & Chemical yield [\%] \\
\hline [Fe(HBED-CC-TFP $)]$ & 37 \\
\hline $\mathrm{PEG}_{2}-[\mathrm{Fe}(\mathrm{HBED}-\mathrm{CC}$-propargylamine $)]$ & 43 \\
\hline Glu-urea-Lys([Fe(HBED-CC-propargylamine)]) & 36 \\
\hline Glu-urea-Lys $\left((\mathrm{HE})_{1}-\mathrm{CO}\left(\mathrm{CH}_{2}\right)_{4}-\mathrm{N}_{3}\right)$ & 66 \\
\hline Glu-urea-Lys $\left((\mathrm{HE})_{3}-\mathrm{CO}\left(\mathrm{CH}_{2}\right)_{4}-\mathrm{N}_{3}\right)$ & 13 \\
\hline Glu-urea-Lys $\left((\mathrm{WE})_{1}-\mathrm{CO}\left(\mathrm{CH}_{2}\right)_{4}-\mathrm{N}_{3}\right)$ & 58 \\
\hline $\mathrm{PEG}_{2}-(\mathrm{EH})_{3}-\mathrm{CO}\left(\mathrm{CH}_{2}\right)_{4}-\mathrm{N}_{3}$ & 35 \\
\hline Glu-urea-Lys $\left((\mathrm{HE})_{1}-\mathrm{HBED}-\mathrm{CC}-\mathrm{PEG}_{2}-\mathrm{NH}_{2}\right)$ & 15 \\
\hline Glu-urea-Lys $\left((\mathrm{HE})_{3}-\mathrm{HBED}-\mathrm{CC}-\mathrm{PEG}_{2}-\mathrm{NH}_{2}\right)$ & 23 \\
\hline Glu-urea-Lys $\left((\mathrm{WE})_{1}\right.$-HBED-CC-PEG $\left.2-\mathrm{NH}_{2}\right)$ & 16 \\
\hline Glu-urea-Lys ([Fe(HBED-CC-(HE) $\left.\left.\left.)_{3}-\mathrm{PEG}_{2}-\mathrm{NH}_{2}\right)\right]\right)$ & 13 \\
\hline
\end{tabular}


Supplemental Table 3. Organ distribution of $0.06 \mathrm{nmol}{ }^{68} \mathrm{Ga}-$ labeled compounds in LNCaP-tumor bearing BALB/c nu/nu mice 1 h p.i.*.

\begin{tabular}{|c|c|c|c|c|}
\hline & $\begin{array}{c}{ }^{68} \text { Ga-Glu-urea-Lys- } \\
\text { (HE)1-HBED-CC- } \\
\text { IRDye800CW } \\
{[\% \text { ID/g] }}\end{array}$ & $\begin{array}{c}{ }^{68} \text { Ga-Glu-urea-Lys- } \\
\text { (WE)1-HBED-CC- } \\
\text { IRDye800CW } \\
{[\% \text { ID/g] }}\end{array}$ & $\begin{array}{c}{ }^{68} \mathrm{Ga}-\text { Glu-urea-Lys- } \\
\text { (HE)3-HBED-CC- } \\
\text { IRDye800CW } \\
{[\% / D / g]}\end{array}$ & $\begin{array}{c}{ }^{68} \text { Ga-Glu-urea-Lys- } \\
\text { HBED-CC-(HE) }{ }^{-} \\
\text {IRDye800CW } \\
{[\% / D / g]}\end{array}$ \\
\hline Blood & $1.52 \pm 0.64$ & $1.61 \pm 0.55$ & $1.12 \pm 0.64$ & $0.80 \pm 0.31$ \\
\hline Heart & $0.94 \pm 0.14$ & $1.49 \pm 0.51$ & $1.36 \pm 0.37$ & $0.57 \pm 0.26$ \\
\hline Lung & $2.46 \pm 0.46$ & $2.57 \pm 1.01$ & $2.34 \pm 0.37$ & $1.78 \pm 1.19$ \\
\hline Spleen & $8.17 \pm 0.23$ & $12.94 \pm 2.00$ & $3.47 \pm 1.39$ & $8.88 \pm 2.93$ \\
\hline Liver & $1.19 \pm 0.30$ & $1.73 \pm 0.61$ & $1.25 \pm 0.18$ & $0.72 \pm 0.05$ \\
\hline Kidney & $84.34 \pm 2.13$ & $104.58 \pm 20.79$ & $109.27 \pm 10.33$ & $92.54 \pm 21.07$ \\
\hline Muscle & $1.03 \pm 0.34$ & $1.00 \pm 0.37$ & $1.18 \pm 0.15$ & $0.97 \pm 0.38$ \\
\hline Intestine & $1.29 \pm 0.36$ & $0.96 \pm 0.37$ & $1.31 \pm 0.00$ & $0.65 \pm 0.21$ \\
\hline Brain & $0.30 \pm 0.10$ & $0.15 \pm 0.04$ & $0.18 \pm 0.02$ & $0.18 \pm 0.06$ \\
\hline Tumor & $3.92 \pm 0.31$ & $3.85 \pm 1.10$ & $7.59 \pm 0.95$ & $3.32 \pm 1.51$ \\
\hline
\end{tabular}

Supplemental Table 4. Tumor-to-organ ratios of $0.06 \mathrm{nmol}{ }^{68} \mathrm{Ga}$-labeled compounds in LNCaP-tumor bearing BALB/c nu/nu mice 1 h p.i.*.

\begin{tabular}{l|cccc} 
& $\begin{array}{c}{ }^{68} \text { Ga-Glu-urea-Lys- } \\
\text { (HE)1-HBED-CC- } \\
\text { IRDye800CW }\end{array}$ & $\begin{array}{c}{ }^{68} \text { Ga-Glu-urea-Lys- } \\
\text { (WE)1-HBED-CC- } \\
\text { IRDye800CW }\end{array}$ & $\begin{array}{c}{ }^{68} \text { Ga-Glu-urea-Lys- } \\
\text { (HE) }-H B E D-C C- \\
\text { IRDye800CW }\end{array}$ & $\begin{array}{c}{ }^{68 G a-G l u-u r e a-L y s-~} \\
\text { HBED-CC-(HE) }\end{array}$ \\
IRDye800CW
\end{tabular}

* Data are expressed as mean tumor-to-organ ratio \pm SD $(n=3)$. 
Supplemental Table 5. Organ distribution of $0.06 \mathrm{nmol}{ }^{68} \mathrm{Ga}-$ labeled Glu-urea-Lys-(HE) 3 -HBED-CCIRDye800CW in PC-3-tumor bearing BALB/c nu/nu mice $1 \mathrm{~h}$ p.i.*.

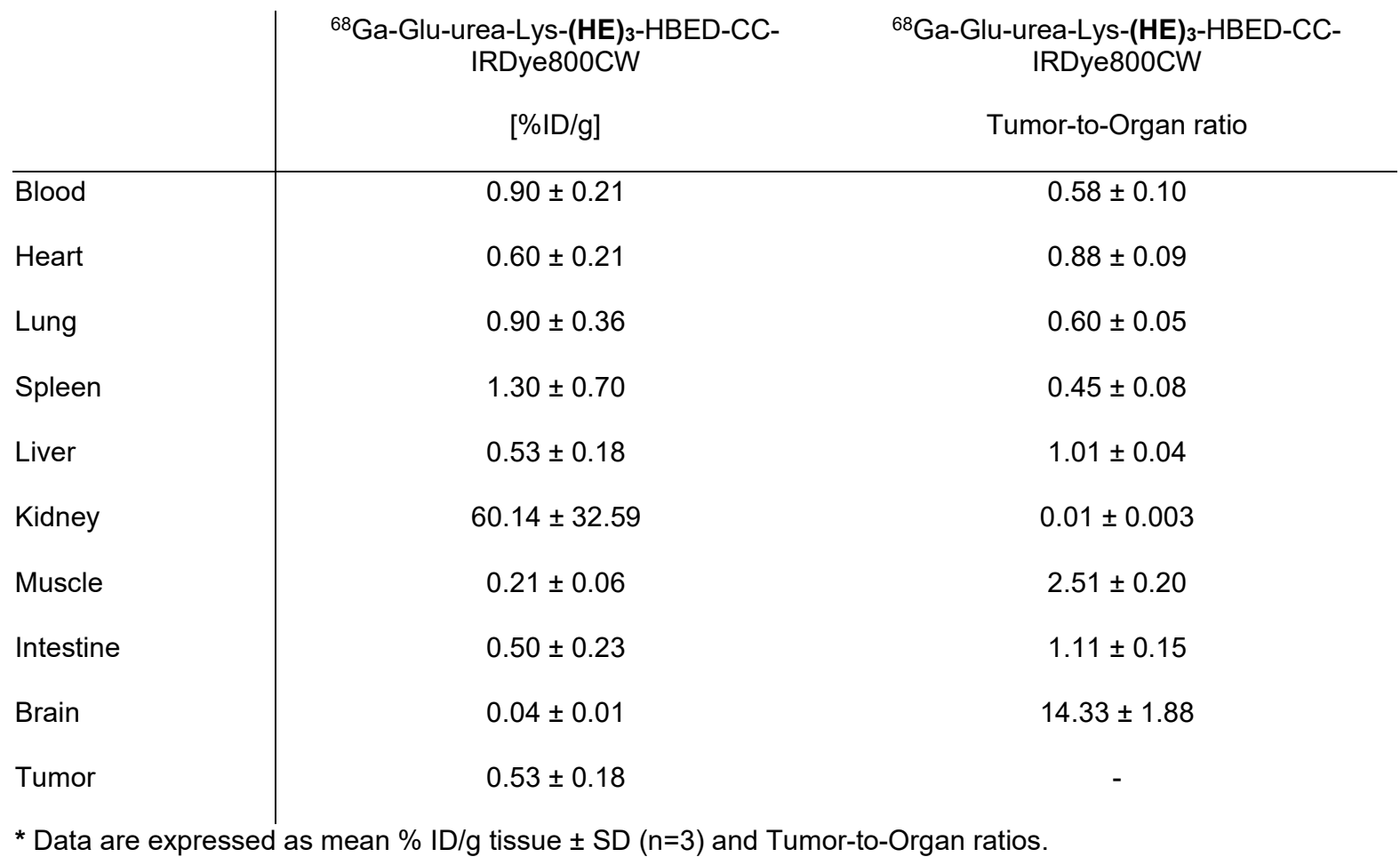

Supplemental Table 6. Organ distribution of $0.06 \mathrm{nmol}{ }^{68} \mathrm{Ga}$-labeled Glu-urea-Lys-(HE) 3 -HBED-CCIRDye800CW in LNCaP-tumor bearing BALB/c nu/nu mice 2 h p.i.*.

\begin{tabular}{|c|c|c|}
\hline & $\begin{array}{c}{ }^{68} \mathrm{Ga}-\mathrm{Glu}-\text { urea-Lys-(HE) } 3-H B E D-C C- \\
\text { IRDye800CW } \\
{[\% \mathrm{ID} / \mathrm{g}]}\end{array}$ & $\begin{array}{c}{ }^{68} \mathrm{Ga}-\text { Glu-urea-Lys-(HE)3-HBED-CC } \\
\text { IRDye800CW } \\
\text { Tumor-to-Organ ratio }\end{array}$ \\
\hline Blood & $0.65 \pm 0.01$ & $4.73 \pm 1.42$ \\
\hline Heart & $0.53 \pm 0.08$ & $5.84 \pm 1.45$ \\
\hline Lung & $0.89 \pm 0.05$ & $3.43 \pm 0.89$ \\
\hline Spleen & $0.76 \pm 0.16$ & $4.02 \pm 0.70$ \\
\hline Liver & $0.76 \pm 0.11$ & $4.01 \pm 0.77$ \\
\hline Kidney & $55.81 \pm 9.76$ & $0.06 \pm 0.01$ \\
\hline Muscle & $0.47 \pm 0.11$ & $6.50 \pm 0.95$ \\
\hline Intestine & $0.67 \pm 0.17$ & $4.66 \pm 1.05$ \\
\hline Brain & $0.23 \pm 0.15$ & $14.83 \pm 3.24$ \\
\hline Tumor & $3.10 \pm 1.17$ & - \\
\hline
\end{tabular}


Supplemental Table 7. Organ distribution of $0.5 \mathrm{nmol}{ }^{68} \mathrm{Ga}-$-labeled Glu-urea-Lys-(HE) 3 -HBED-CC-IRDye800CW in LNCaP-tumor bearing BALB/c nu/nu mice $1 \mathrm{~h}$ p.i.*.

\begin{tabular}{|c|c|c|}
\hline & $\begin{array}{c}{ }^{68} \text { Ga-Glu-urea-Lys-(HE)3-HBED-CC- } \\
\text { IRDye800CW } \\
{[\% \text { ID/g] }}\end{array}$ & $\begin{array}{c}{ }^{68} \mathrm{Ga}-\mathrm{Glu} \text {-urea-Lys-(HE) } 3 \text {-HBED-CC } \\
\text { IRDye800CW } \\
\text { Tumor-to-Organ ratio }\end{array}$ \\
\hline Blood & $3.10 \pm 1.40$ & $6.89 \pm 0.62$ \\
\hline Heart & $2.64 \pm 0.59$ & $7.87 \pm 0.60$ \\
\hline Lung & $4.27 \pm 1.02$ & $4.96 \pm 0.95$ \\
\hline Spleen & $6.90 \pm 3.21$ & $3.15 \pm 0.62$ \\
\hline Liver & $2.43 \pm 0.62$ & $8.55 \pm 0.61$ \\
\hline Kidney & $229.08 \pm 53.38$ & $0.09 \pm 0.01$ \\
\hline Muscle & $1.17 \pm 0.13$ & $17.84 \pm 2.26$ \\
\hline Intestine & $2.66 \pm 0.89$ & $7.89 \pm 0.32$ \\
\hline Brain & $0.15 \pm 0.06$ & $144.46 \pm 27.52$ \\
\hline Tumor & $20.98 \pm 6.54$ & - \\
\hline
\end{tabular}

Supplemental Table 8. Organ distribution of $0.5 \mathrm{nmol}{ }^{68} \mathrm{Ga}$-labeled Glu-urea-Lys-(HE) 3 -HBED-CC-IRDye800CW in LNCaP-tumor bearing BALB/c nu/nu mice $2 \mathrm{~h}$ p.i.*.

\begin{tabular}{|c|c|c|}
\hline & $\begin{array}{c}{ }^{68} \mathrm{Ga}-\text { Glu-urea-Lys-(HE)3-HBED-CC- } \\
\text { IRDye800CW } \\
{[\% \mathrm{ID} / \mathrm{g}]}\end{array}$ & $\begin{array}{c}{ }^{68} \text { Ga-Glu-urea-Lys-(HE)3-HBED-CC- } \\
\text { IRDye800CW } \\
\text { Tumor-to-Organ ratio }\end{array}$ \\
\hline Blood & $1.91 \pm 0.84$ & $6.56 \pm 0.98$ \\
\hline Heart & $1.34 \pm 0.73$ & $9.45 \pm 0.72$ \\
\hline Lung & $2.07 \pm 0.89$ & $6.04 \pm 0.94$ \\
\hline Spleen & $2.26 \pm 1.16$ & $5.57 \pm 0.50$ \\
\hline Liver & $1.62 \pm 0.86$ & $7.79 \pm 0.75$ \\
\hline Kidney & $176.10 \pm 97.86$ & $0.07 \pm 0.01$ \\
\hline Muscle & $0.56 \pm 0.37$ & $23.08 \pm 0.81$ \\
\hline Intestine & $1.30 \pm 0.56$ & $9.91 \pm 2.18$ \\
\hline Brain & $0.09 \pm 0.05$ & $138.96 \pm 25.36$ \\
\hline Tumor & $12.90 \pm 8.46$ & - \\
\hline
\end{tabular}




\section{References}

1. Schafer M, Bauder-Wust $U$, Leotta $K$, et al. A dimerized urea-based inhibitor of the prostatespecific membrane antigen for 68Ga-PET imaging of prostate cancer. EJNMMI Res. 2012;2:23.

2. Eder $M$, Wangler $B$, Knackmuss $S$, et al. Tetrafluorophenolate of HBED-CC: a versatile conjugation agent for 68Ga-labeled small recombinant antibodies. Eur $\mathrm{J} \mathrm{Nucl}$ Med $\mathrm{Mol}$ Imaging. 2008;35:1878-1886. 